\title{
HOLONOMY PERTURBATIONS OF THE CHERN-SIMONS FUNCTIONAL FOR LENS SPACES
}

\author{
DAVID BOOZER
}

\begin{abstract}
We describe a scheme for constructing generating sets for Kronheimer and Mrowka's singular instanton knot homology for the case of knots in lens spaces. The scheme involves Heegaard-splitting a lens space containing a knot into two solid tori. One solid torus contains a portion of the knot consisting of an unknotted arc, as well as holonomy perturbations of the Chern-Simons functional used to define the homology theory. The other solid torus contains the remainder of the knot. The Heegaard splitting yields a pair of Lagrangians in the traceless $S U(2)$-character variety of the twice-punctured torus, and the intersection points of these Lagrangians comprise the generating set that we seek. We illustrate the scheme by constructing generating sets for several example knots. Our scheme is a direct generalization of a scheme introduced by Hedden, Herald, and Kirk for describing generating sets for knots in $S^{3}$ in terms of Lagrangian intersections in the traceless $S U(2)$-character variety for the 2 -sphere with four punctures.
\end{abstract}

\section{CONTEnTS}

1. Introduction

2. Character varieties

2.1. The character variety $R\left(T^{2}, 2\right)$

2.2. The character variety $R\left(S^{1} \times D^{2}, A_{1}\right)$

2.3. The character variety $R^{\natural}\left(S^{1} \times D^{2}, A_{1}\right)$

2.4. The character variety $R_{\pi}^{\natural}\left(S^{1} \times D^{2}, A_{1}\right)$

3. The group $\mathrm{MCG}_{2}\left(T^{2}\right)$ and its action on $R\left(T^{2}, 2\right)$

3.1. The mapping class group $\mathrm{MCG}_{2}\left(T^{2}\right)$ for the twice-punctured torus

3.2. The Birman sequence for the twice-punctured torus

3.3. The action of $\mathrm{MCG}_{2}\left(T^{2}\right)$ on $R\left(T^{2}, 2\right)$

4. Nondegeneracy

4.1. Constrained group cohomology

4.2. Regularity

4.3. Smoothness

4.4. Transversality

5. Examples

5.1. Trefoil in $S^{3}$

5.2. Unknot in $L(p, 1)$ for $p \bmod 4 \neq 0$

5.3. Simple knot in $L(p, 1)$ in homology class $1 \in \mathbb{Z}_{p}=H_{1}(L(p, 1) ; \mathbb{Z})$

Acknowledgments

References

\section{INTRODUCTION}

Singular instanton homology was introduced by Kronheimer and Mrowka to describe knots in 3-manifolds 15, 16, 17. Singular instanton homology is defined in terms of gauge theory, but has important implications for Khovanov homology, a knot homology theory that categorifies the Jones polynomial and that can be defined in a purely combinatorial fashion. Specifically, given a knot $K$ in $S^{3}$, Kronheimer and Mrowka showed that there is a spectral sequence whose $E_{2}$ page is the reduced Khovanov homology of the mirror knot

Date: November 6, 2018. 
$\bar{K}$, and that converges to the singular instanton homology of $K$. Using this spectral sequence, Kronheimer and Mrowka proved a key property of Khovanov homology: a knot in $S^{3}$ is the unknot if and only if its reduced Khovanov homology has rank 1 . This result is obtained by proving the analogous result for singular instanton homology and then using the rank inequality implied by the spectral sequence.

Calculations of singular instanton homology are generally difficult to carry out, though some results are known. For example, Kronheimer and Mrowka showed that the singular instanton homology of an alternating knot in $S^{3}$ is isomorphic to the reduced Khovanov homology of its mirror (modulo grading), since for such knots the spectral sequence collapses at the $E_{2}$ page. In recent work, Hedden, Herald, and Kirk have described a scheme for producing generating sets for singular instanton homology for a variety of knots in $S^{3}$, which can sometimes be used in conjunction with the spectral sequence to compute the singular instanton homology itself [12. Their scheme works as follows.

Singular instanton homology is defined in terms of the Morse complex of a perturbed Chern-Simons functional. The unperturbed Chern-Simons functional is typically not Morse, so to obtain a well-defined homology theory it is necessary to include a small perturbation term. For the case of knots in $S^{3}$, Hedden, Herald, and Kirk show how a suitable perturbation can be constructed. Their scheme involves Heegaardsplitting $S^{3}$ into a pair of solid 3-balls $B_{1}$ and $B_{2}$. The ball $B_{1}$ contains a portion of the knot $K$ consisting of two unknotted arcs, together with a specific holonomy perturbation of the Chern-Simons functional. The ball $B_{2}$ contains the remainder of the knot. The Heegaard splitting of $S^{3}$ yields a pair of Lagrangians $L_{1}$ and $L_{2}$ in the traceless $S U(2)$-character variety of the 2 -sphere with four punctures $R\left(S^{2}, 4\right)$, a symplectic manifold known as the pillowcase that is homeomorphic to the 2-sphere. Specifically, the Lagrangians $L_{1}$ and $L_{2}$ describe conjugacy classes of $S U(2)$-representations of the fundamental group of the 2-sphere with four punctures that extend to $B_{1}-K$ and $B_{2}-K$, respectively. In many cases, the points of intersection of $L_{1}$ and $L_{2}$ constitute a generating set for singular instanton homology. The essential idea of the scheme is to confine all of the perturbation data to a standard ball $B_{1}$ corresponding to a Lagrangian $L_{1}$ that can be described explicitly. The problem of constructing a generating set for a particular knot thus reduces to describing the Lagrangian $L_{2}$, a task that is facilitated by the fact that the Chern-Simons functional is unperturbed on the ball $B_{2}$. In further work, Hedden, Herald, and Kirk define pillowcase homology to be the Lagrangian Floer homology of the pair $\left(L_{1}, L_{2}\right)$ [13. They conjecture that pillowcase homology is isomorphic to singular instanton homology and compute some examples that support this conjecture.

In the present paper we generalize the scheme of Hedden, Herald, and Kirk to the case of knots in lens spaces. We Heegaard-split a lens space $Y$ containing a knot $K$ into two solid tori $U_{1}$ and $U_{2}$. The solid torus $U_{1}$ contains a portion of the knot consisting of an unknotted arc $A_{1}$, together with a specific holonomy perturbation. The solid torus $U_{2}$ contains the remainder of the knot. From the Heegaard splitting of $Y$ we obtain a pair of Lagrangians $L_{1}$ and $L_{2}$ in the traceless $S U(2)$-character variety of the twice-punctured torus $R\left(T^{2}, 2\right)$, and in many cases the points of intersection of $L_{1}$ and $L_{2}$ constitute a generating set.

To explain the details of our scheme, we must first define several character varieties. Critical points of the unperturbed Chern-Simons functional are flat connections. Gauge-equivalence classes of such flat connections correspond to conjugacy classes of homomorphisms $\rho: \pi_{1}(Y-K \cup H \cup W) \rightarrow S U(2)$, where $H$ is a small loop around $K$ and $W$ is an arc connecting $K$ to $H$, as shown in Figure 1, and the homomorphisms are required to take loops around $K$ and $H$ to traceless matrices and loops around $W$ to -1 . The space of such conjugacy classes form a character variety that we will denote by $R^{\natural}(Y, K)$. The conditions on $\rho$ involving $H$ and $W$ are imposed in order to avoid reducible connections. It will also be useful to define a character variety $R(Y, K)$ in which we do not impose these conditions, and which consists of conjugacy classes of homomorphisms $\rho: \pi_{1}(Y-K) \rightarrow S U(2)$ that take loops around $K$ to traceless matrices.

The character variety $R^{\natural}(Y, K)$ is typically degenerate, in which case the unperturbed Chern-Simons functional is not Morse. We can render the Chern-Simons functional Morse by introducing a suitable holonomy perturbation term that vanishes outside of a solid torus obtained by thickening a loop $P \subset Y$. The net effect of the perturbation is to modify the corresponding character variety: the critical points of the perturbed Chern-Simons functional correspond to conjugacy classes of homomorphisms $\rho: \pi_{1}(Y-K \cup$ $H \cup W \cup P) \rightarrow S U(2)$, where $\rho$ obeys the same conditions as for $R^{\natural}(Y, K)$ as well as an additional condition involving the loop $P$ that we will describe in Section 2.4. We will denote the character variety corresponding to the perturbed Chern-Simons functional by $R_{\pi}^{\natural}(Y, K)$. 


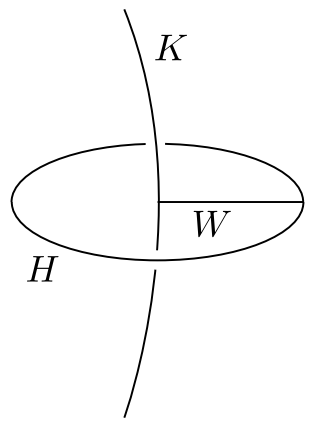

Figure $1 . \quad$ The knot $K$, loop $H$, and $\operatorname{arc} W$.

Example 1.1. For the trefoil $K$ in $S^{3}$, one can show that

$$
R\left(S^{3}, K\right)=\{2 \text { points }\}, \quad R^{\natural}\left(S^{3}, K\right)=\{1 \text { point }\} \amalg S^{1}, \quad R_{\pi}^{\natural}\left(S^{3}, K\right)=\{3 \text { points }\},
$$

where the perturbation used to define $R_{\pi}^{\natural}\left(S^{3}, K\right)$ is as described in Section 5.1 .

Our goal, then, is to devise an effective means of calculating $R_{\pi}^{\natural}(Y, K)$. We Heegaard-split $Y$ along a torus that transversely intersects the knot $K$ in two points, resulting in two solid tori $U_{1}, U_{2} \subset Y$. We define arcs $A_{i}=U_{i} \cap K$ that correspond to the portion of the knot $K$ contained in the solid torus $U_{i}$, and we assume that the $\operatorname{arc} A_{1}$ is unknotted. We further assume that the loop $H$ and $\operatorname{arc} W$, as well as the holonomy perturbation described by the loop $P$, are all contained in the solid torus $U_{1}$. Specifically, we choose the loop $P$ as shown in Figure 6. We define character varieties $R_{\pi}^{\natural}\left(U_{1}, A_{1}\right)$ and $R\left(U_{2}, A_{2}\right)$ in analogy with $R_{\pi}^{\natural}(Y, K)$ and $R(Y, K)$ : the character variety $R_{\pi}^{\natural}\left(U_{1}, A_{1}\right)$ consists of conjugacy classes of homomorphisms $\rho: \pi_{1}\left(U_{1}-A_{1} \cup H \cup W \cup P\right) \rightarrow S U(2)$ that take loops around $A_{1}$ and $H$ to traceless matrices and loops around $W$ to -1 , and satisfy an additional requirement involving $P$ as described in Section 2.4. and $R\left(U_{2}, A_{2}\right)$ consists of conjugacy classes of homomorphisms $\rho: \pi_{1}\left(U_{2}-A_{2}\right) \rightarrow S U(2)$ that take loops around $A_{2}$ to traceless matrices. We also define a character variety $R\left(T^{2}, 2\right)$ for the torus with two punctures $T^{2}-\left\{p_{1}, p_{2}\right\}$, which consists of conjugacy classes of homomorphisms $\rho: \pi_{1}\left(T^{2}-\left\{p_{1}, p_{2}\right\}\right) \rightarrow S U(2)$ that take loops around $p_{1}$ and $p_{2}$ to traceless matrices.

We pick homeomorphisms $\phi_{i}: S^{1} \times D^{2} \rightarrow U_{i}$ from the standard solid torus $S^{1} \times D^{2}$ to $U_{i}$, and define homeomorphisms $\partial \phi_{i}: T^{2} \rightarrow U_{1} \cap U_{2}$ by restricting the domain and codomain of $\phi_{i}$. For simplicity, we denote the preimage of $A_{i}$ under $\phi_{i}$ by $A_{i}$, and the preimages of $H, W$, and $P$ under $\phi_{1}$ by $H, W$, and $P$. We can pull back along the composition of $\partial \phi_{1}$ with the inclusions $U_{1} \cap U_{2} \rightarrow U_{i}$ to obtain maps $R_{\pi}^{\natural}\left(U_{1}, A_{1}\right) \rightarrow R\left(T^{2}, 2\right)$ and $R\left(U_{2}, A_{2}\right) \rightarrow R\left(T^{2}, 2\right)$, and we can pull back along the inclusions $U_{i} \rightarrow Y$ to obtain maps $R_{\pi}^{\natural}(Y, K) \rightarrow R_{\pi}^{\natural}\left(U_{1}, A_{1}\right)$ and $R_{\pi}^{\natural}(Y, K) \rightarrow R\left(U_{2}, A_{2}\right)$. We have a commutative diagram:

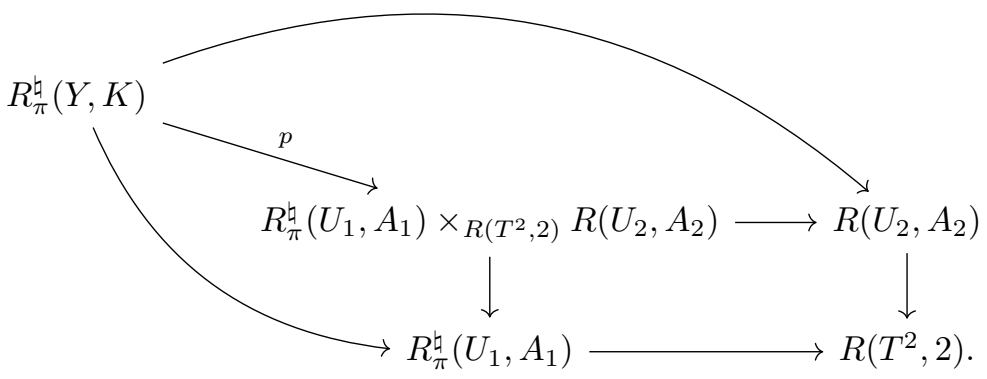

Here $p$ is an induced map from $R_{\pi}^{\natural}(Y, K)$ to the fiber product $R_{\pi}^{\natural}\left(U_{1}, A_{1}\right) \times_{R\left(T^{2}, 2\right)} R\left(U_{2}, A_{2}\right)$. We want to use diagram (2) to describe $R_{\pi}^{\natural}(Y, K)$ in terms of the intersection of Lagrangians in $R\left(T^{2}, 2\right)$. Our first task is to obtain an explicit description of the character variety $R\left(T^{2}, 2\right)$ that generalizes the pillowcase. We prove:

Theorem 1.1. The character variety $R\left(T^{2}, 2\right)$ is the union of two pieces $P_{4}$ and $P_{3}$, where $P_{4}$ is homeomorphic to $S^{2} \times S^{2}-\Delta$ and $\Delta=\{(\hat{r}, \hat{r})\}$ is the diagonal, and $P_{3}$ deformation retracts onto the pillowcase. (The spaces $P_{4}$ and $P_{3}$ are described in Theorems 2.2 and 2.3.) 
Our next task is to understand the images of $R_{\pi}^{\natural}\left(U_{1}, A_{1}\right)$ and $R\left(U_{2}, A_{2}\right)$ in $R\left(T^{2}, 2\right)$, which we will denote by $L_{1}$ and $L_{2}$. We pull back along the homeomorphisms $\phi_{i}: S^{1} \times D^{2} \rightarrow U_{i}$ to define isomorphisms from $R_{\pi}^{\natural}\left(U_{1}, A_{1}\right)$ and $R\left(U_{2}, A_{2}\right)$ to the corresponding character varieties $R_{\pi}^{\natural}\left(S^{1} \times D^{2}, A_{1}\right)$ and $R\left(S^{1} \times D^{2}, A_{2}\right)$ for the standard solid torus $S^{1} \times D^{2}$. We pull back along the inclusion $T^{2} \rightarrow S^{1} \times D^{2}$ to define maps $R\left(S^{1} \times D^{2}, A_{1}\right) \rightarrow R\left(T^{2}, 2\right), R_{\pi}^{\natural}\left(S^{1} \times D^{2}, A_{1}\right) \rightarrow R\left(T^{2}, 2\right)$ and $R\left(S^{1} \times D^{2}, A_{2}\right) \rightarrow R\left(T^{2}, 2\right)$, and we define $L_{d}, L_{s}$, and $L_{2}^{\prime}$ to be the images of these maps. We refer to $L_{d}$ as the disk Lagrangian and $L_{s}$ as the sphere Lagrangian since, as we will see, $R\left(S^{1} \times D^{2}, A_{1}\right)$ is homeomorphic to a disk and $R_{\pi}^{\natural}\left(S^{1} \times D^{2}, A_{1}\right)$ is homeomorphic to a sphere. The mapping class group $\mathrm{MCG}_{2}\left(T^{2}\right)$ of the twice-punctured torus acts on the space $R\left(T^{2}, 2\right)$ from the right in a way that we explicitly characterize in Section 3 . We find that the images of $R_{\pi}^{\natural}\left(U_{1}, A_{1}\right)$ and $R\left(U_{2}, A_{2}\right)$ in $R\left(T^{2}, 2\right)$ are $L_{1}=L_{s}$ and $L_{2}=L_{2}^{\prime} \cdot f$, where $f=\left[\partial \phi_{2}^{-1} \circ \partial \phi_{1}\right] \in \mathrm{MCG}_{2}\left(T^{2}\right)$. We prove the following results:

Theorem 1.2. The space $R\left(S^{1} \times D^{2}, A_{1}\right)$ is homeomorphic to the closed disk $D^{2}$, and the map $R\left(S^{1} \times\right.$ $\left.D^{2}, A_{1}\right) \rightarrow R\left(T^{2}, 2\right)$ is injective. (An explicit parameterization of the image $L_{d}$ of the map is given in Theorem 2.4.

Theorem 1.3. The space $R_{\pi}^{\natural}\left(S^{1} \times D^{2}, A_{1}\right)$ is homeomorphic to $S^{2}$. The map $R_{\pi}^{\natural}\left(S^{1} \times D^{2}, A_{1}\right) \rightarrow R\left(T^{2}, 2\right)$ is injective away from the points of $R_{\pi}^{\natural}\left(S^{1} \times D^{2}, A_{1}\right)$ that correspond to the north and south pole of $S^{2}$, which are mapped to the same point. All of the representations in the image $L_{s}$ of the map are nonabelian. (An explicit parameterization of $L_{s}$ is given in Theorems 2.9 and 2.10.)

Corollary 1.1. The map $p: R_{\pi}^{\natural}(Y, K) \rightarrow R_{\pi}^{\natural}\left(U_{1}, A_{1}\right) \times_{R\left(T^{2}, 2\right)} R\left(U_{2}, A_{2}\right)$ in diagram 22 is injective.

Proof. Consider a point $\left(\left[\rho_{1}\right],\left[\rho_{2}\right]\right)$ in $R_{\pi}^{\natural}\left(U_{1}, A_{1}\right) \times_{R\left(T^{2}, 2\right)} R\left(U_{2}, A_{2}\right)$, so $\rho_{1}$ and $\rho_{2}$ pull back to the same homomorphism $\rho_{12}: \pi_{1}\left(T^{2}-\left\{p_{1}, p_{2}\right\}\right) \rightarrow S U(2)$. One can show (see [12] Lemma 4.2) that the fiber $p^{-1}\left(\left[\rho_{1}\right],\left[\rho_{2}\right]\right)$ is homeomorphic to the double coset space $\operatorname{Stab}\left(\rho_{1}\right) \backslash \operatorname{Stab}\left(\rho_{12}\right) / \operatorname{Stab}\left(\rho_{2}\right)$, where

$$
\operatorname{Stab}(\rho)=\left\{g \in S U(2) \mid g \rho(x) g^{-1}=\rho(x) \text { for all } x \text { in the domain of } \rho\right\} .
$$

The center of $S U(2)$ is $Z(S U(2))=\{ \pm 1\}$. By Theorem 1.3 we have that $\operatorname{Stab}\left(\rho_{12}\right)=Z(S U(2))$, and $Z(S U(2)) \subseteq \operatorname{Stab}\left(\rho_{i}\right) \subseteq \operatorname{Stab}\left(\rho_{12}\right)$, so $\operatorname{Stab}\left(\rho_{i}\right)=\operatorname{Stab}\left(\rho_{12}\right)=Z(S U(2))$ and thus the fibers of $p$ are points.

By introducing a suitable perturbation we obtain a finite character variety $R_{\pi}^{\natural}(Y, K)$, each point of which corresponds to a gauge-orbit of connections that are critical points of the perturbed Chern-Simons functional. In order for $R_{\pi}^{\natural}(Y, K)$ to serve as a generating set for singular instanton homology, each point in $R_{\pi}^{\natural}(Y, K)$ must be nondegenerate; that is, at each connection representing a point in $R_{\pi}^{\natural}(Y, K)$ we want the Hessian of the perturbed Chern-Simons functional to be nondegenerate when restricted to a complement of the tangent space to the gauge-orbit of that connection. We show that there is a simple criterion for determining when a point $R_{\pi}^{\natural}(Y, K)$ is nondegenerate:

Theorem 1.4. Assume that the map $R\left(U_{2}, A_{2}\right) \rightarrow R\left(T^{2}, 2\right)$ is an injective immersion. Consider a point $[\rho] \in L_{s} \cap L_{2} \subset R\left(T^{2}, 2\right)$ that is the image of a regular point of $R\left(U_{2}, A_{2}\right)$ and is not the double-point of $L_{s}$. By Corollary 1.1, the point $[\rho]$ is the image of a unique point $\left[\rho^{\prime}\right] \in R_{\pi}^{\natural}(Y, K)$ under the pullback map $R_{\pi}^{\natural}(Y, K) \rightarrow R\left(T^{2}, 2\right)$. The point $\left[\rho^{\prime}\right]$ is nondegenerate if and only if the intersection of $L_{s}$ with $L_{2}$ at $[\rho]$ is transverse.

Collecting these results, we find if the hypotheses of Theorem 1.4 are satisfied for every point in $L_{s} \cap L_{2}$, then every point in $R_{\pi}^{\natural}(Y, K)$ is nondegenerate and the pullback map $R_{\pi}^{\natural}(Y, K) \rightarrow R\left(T^{2}, 2\right)$ is injective with image $L_{s} \cap L_{2}$. Thus $R_{\pi}^{\natural}(Y, K)$ is a generating set for singular instanton homology consisting of $\left|L_{s} \cap L_{2}\right|$ generators.

In particular, we can use Theorem 1.2 to treat the case of $(1,1)$-knots. By definition, a $(1,1)$-knot is a knot $K$ in a lens space $Y$ that has a Heegaard splitting into a pair of solid tori $U_{1}, U_{2} \subset Y$ such that the components $U_{1} \cap K$ and $U_{2} \cap K$ of the knot in each solid torus are unknotted arcs. It is known that $(1,1)$-knots include torus knots and two-bridge knots. For $(1,1)$-knots, the map $R\left(U_{2}, A_{2}\right) \rightarrow R\left(T^{2}, 2\right)$ is an embedding with image $L_{2}=L_{d} \cdot f$.

In Section 5 we use these results to calculate generating sets for several $(1,1)$-knots. We first rederive known results for knots in $S^{3}$ : we produce generating sets for the unknot (one generator) and trefoil (three generators), which allow us to compute the singular instanton homology for these knots. Next we consider 
knots in lens spaces $L(p, 1)$. We show that the unknot in $L(p, 1)$ has a generating set with $p$ generators for $p \bmod 4 \neq 0$. A knot $K$ in a lens space $L(p, q)$ is said to be simple if the lens space can be Heegaard-split into solid tori $U_{1}$ and $U_{2}$ with meridian disks $D_{1}$ and $D_{2}$ such that $D_{1}$ intersects $D_{2}$ in $p$ points and $K \cap U_{i}$ is an unknotted arc in disk $D_{i}$ for $i=1,2$ (see [11]). Up to isotopy, there is exactly one simple knot in each nonzero homology class of $H_{1}(L(p, q) ; \mathbb{Z})=\mathbb{Z}_{p}$. We prove the following result:

Theorem 1.5. If $K$ is the unique simple knot representing the homology class $1 \in \mathbb{Z}_{p}=H_{1}(L(p, 1) ; \mathbb{Z})$ of the lens space $L(p, 1)$, then the rank of the singular instanton homology of $K$ is at most $p$.

For a simple knot $K$ in the lens space $Y=L(p, q)$, the knot Floer homology $\widehat{H F K}(Y, K)$ has rank $p$ (see [11). Thus, Theorem 1.5 is consistent with Kronheimer and Mrowka's conjecture that for a knot $K$ in a 3-manifold $Y$, the ranks of singular instanton homology and knot Floer homology $\widehat{H F K}(Y, K)$ are the same (see 14 Section 7.9). There is a combinatorial method for computing the Floer homology for arbitrary $(1,1)$-knots in $S^{3}$ (see [9]), and it would be interesting to test Kronheimer and Mrowka's conjecture for such knots by using our results to derive bounds on the rank of the singular instanton homology.

Remark 1.1. In what follows $\sigma_{x}, \sigma_{y}$, and $\sigma_{z}$ are the Pauli spin matrices, defined as

$$
\sigma_{x}=\left(\begin{array}{cc}
0 & 1 \\
1 & 0
\end{array}\right), \quad \sigma_{y}=\left(\begin{array}{cc}
0 & -i \\
i & 0
\end{array}\right), \quad \sigma_{z}=\left(\begin{array}{cc}
1 & 0 \\
0 & -1
\end{array}\right) .
$$

\section{Character varieties}

2.1. The character variety $R\left(T^{2}, 2\right)$. Our first task is to understand the structure of $R\left(T^{2}, 2\right)$, the traceless $S U(2)$-character variety of the twice-punctured torus. In general, we make the following definition:

Definition 2.1. Given a surface $S$ with $n$ distinct marked points $p_{1}, \cdots, p_{n} \in S$, we define the character variety $R(S, n)$ to be the space of conjugacy classes of homomorphisms $\rho: \pi_{1}\left(S-\left\{p_{1}, \cdots, p_{n}\right\}\right) \rightarrow S U(2)$ that take loops around the marked points to traceless $S U(2)$-matrices.

Remark 2.1. The character variety $R(S, n)$ can also be interpreted as the moduli space $M(S, n)$ of rank 2 degree 0 semistable parabolic bundles with $n$ marked points, where the underlying holomorphic vector bundle of the parabolic bundle is required to have trivial determinant bundle. A homeomorphism $M(S, n) \rightarrow R(S, n)$ can be constructed by first describing the complex structure of the parabolic bundle in terms of a flat $S U(2)$-connection with prescribed singularities at the puncture points, and then computing the holonomy of the connection around the generators of $\pi_{1}\left(S-\left\{p_{1}, \cdots, p_{n}\right\}\right)$ to define a homomorphism $\rho: \pi_{1}(S-$ $\left.\left\{p_{1}, \cdots, p_{n}\right\}\right) \rightarrow S U(2)$. Other than serving as a consistency check on some of our results, we will not use this interpretation here.

Remark 2.2. For each element $\lambda \in \pi_{1}\left(S-\left\{p_{1}, \cdots, p_{n}\right\}\right)$ there is a corresponding function $R(S, n) \rightarrow \mathbb{R}$, $[\rho] \mapsto \operatorname{tr}(\rho(\lambda))$. Functions of this form will play an important role in what follows.

Before examining the space $R\left(T^{2}, 2\right)$, we first consider the simpler space $R\left(T^{2}\right):=R\left(T^{2}, 0\right)$, which is known as the pillowcase. We have the following well-known result:

Theorem 2.1. The pillowcase $R\left(T^{2}\right)$ is homeomorphic to $S^{2}$.

Proof. The fundamental group of $T^{2}$ is $\pi_{1}\left(T^{2}\right)=\langle A, B \mid[A, B]=1\rangle$, where $A$ and $B$ are represented by the two fundamental cycles. A homomorphism $\rho: \pi_{1}\left(T^{2}\right) \rightarrow S U(2)$ is uniquely determined by $\rho(A)$ and $\rho(B)$, which for simplicity we will also denote by $A$ and $B$. Since $A$ and $B$ commute, any conjugacy class $[\rho] \in R\left(T^{2}\right)$ has a representative of the form

$$
A=\cos \alpha+i \sigma_{z} \sin \alpha, \quad B=\cos \beta+i \sigma_{z} \sin \beta
$$

for some angles $\alpha$ and $\beta$. These equations are invariant under the replacements $\alpha \rightarrow \alpha+2 \pi$ and $\beta \rightarrow \beta+2 \pi$, and we are free to conjugate by $i \sigma_{x}$, so we obtain the following identifications:

$$
(\alpha, \beta) \sim(\alpha+2 \pi, \beta), \quad(\alpha, \beta) \sim(\alpha, \beta+2 \pi), \quad(\alpha, \beta) \sim(-\alpha,-\beta) .
$$

We can thus restrict to a fundamental domain in which $(\alpha, \beta) \in[0,2 \pi] \times[0, \pi]$, with edges identified as shown in Figure 2. From Figure 2 it is clear that this space is homeomorphic to $S^{2}$.

Definition 2.2. We will refer to the four points $[(A, B)]=[( \pm 1, \pm 1)] \in R\left(T^{2}\right)$ as pillowcase points. 


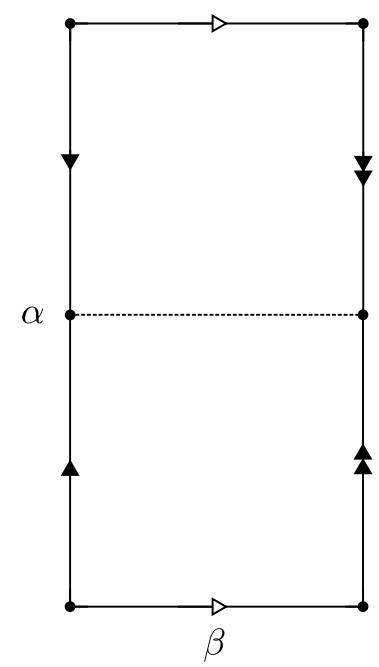

Figure 2. The pillowcase $R\left(T^{2}\right)$. The black dots indicate the four pillowcase points.

Remark 2.3. Theorem 2.1 is consistent with the fact that, as noted in Remark 2.1, $R\left(T^{2}\right)$ is homeomorphic to the moduli space of semistable parabolic bundles $M\left(T^{2}\right):=M\left(T^{2}, 0\right)$, which is known to have the structure of an algebraic variety isomorphic to $\mathbb{C P}^{1}$.

Remark 2.4. One can show that the character variety $R\left(S^{2}, 4\right)$ is also described by a rectangle with edges identified as shown in Figure 2 (see, for example, [12] Section 3.1), so both $R\left(T^{2}\right)$ and $R\left(S^{2}, 4\right)$ are referred to as the pillowcase.

We now consider the space $R\left(T^{2}, 2\right)$. The fundamental group of the twice-punctured torus is

$$
\pi_{1}\left(T^{2}-\left\{p_{1}, p_{2}\right\}\right)=\langle A, B, a, b \mid[A, B] a b=1\rangle,
$$

where $p_{1}$ and $p_{2}$ denote the puncture points, $A$ and $B$ denote the fundamental cycles of the torus, and $a$ and $b$ denote loops around the punctures $p_{1}$ and $p_{2}$, as shown in Figure 3 . As above, we will be sloppy and use the same notation for generators of the fundamental group and their images under $\rho$; for example, we denote $\rho(A)$ by $A$. A homomorphism $\rho: \pi_{1}\left(T^{2}-\left\{p_{1}, p_{2}\right\}\right) \rightarrow S U(2)$ is thus specified by $S U(2)$-matrices $(A, B, a, b)$ such that $a$ and $b$ are traceless and $[A, B]=(a b)^{-1}$.

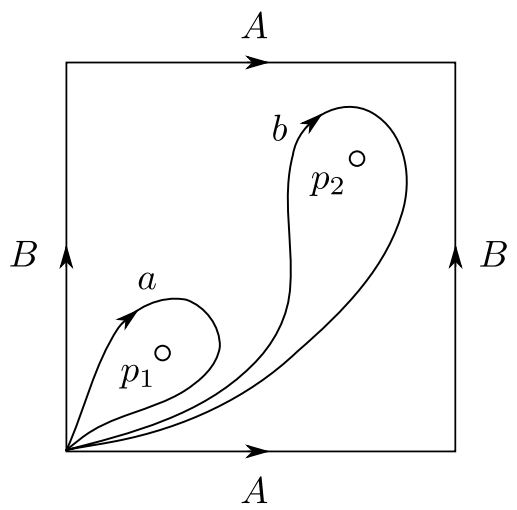

Figure 3. Cycles corresponding to the generators $A, B, a, b$ of the fundamental group $\pi_{1}\left(T^{2}-\left\{p_{1}, p_{2}\right\}\right)$.

The structure of the space $R\left(T^{2}, 2\right)$ can be understood by considering the fibers of a map $\mu: R\left(T^{2}, 2\right) \rightarrow$ $[-1,1]$ of the form described in Remark 2.2 . 
Definition 2.3. We define a map $\mu: R\left(T^{2}, 2\right) \rightarrow[-1,1]$ by

$$
\mu([\rho])=(1 / 2) \operatorname{tr}([A, B])=(1 / 2) \operatorname{tr}\left((a b)^{-1}\right) .
$$

In particular, it is convenient to decompose $R\left(T^{2}, 2\right)$ into the disjoint union of an open piece $P_{4}=$ $\mu^{-1}([-1,1))$ and a closed piece $P_{3}=\mu^{-1}(1)$, which we consider separately. The notation for these pieces is motivated by the fact that, as we will see, the piece $P_{4}$ is four-dimensional and the piece $P_{3}$ is threedimensional.

2.1.1. The piece $P_{4} \subset R\left(T^{2}, 2\right)$. We define the piece $P_{4} \subset R\left(T^{2}, 2\right)$ to be the set of conjugacy classes $[\rho] \in R\left(T^{2}, 2\right)$ such that $\mu([\rho]) \in[-1,1)$. Any conjugacy class $[\rho] \in P_{4}$ has a unique representative $\rho$ for which the matrices $A$ and $B$ have the form

$$
A=r \cos \alpha+i \sigma_{x} \sqrt{1-r^{2}}+i \sigma_{z} r \sin \alpha, \quad B=\cos \beta+i \sigma_{z} \sin \beta,
$$

where $\alpha \in[0,2 \pi], \beta \in(0, \pi)$, and $r \in[0,1)$. To see this, note that given an arbitrary representative we can first conjugate so that the coefficients of $i \sigma_{x}$ and $i \sigma_{y}$ in $B$ are zero and the coefficient of $i \sigma_{z}$ is positive, and then rotate about the $z$-axis so the coefficient of $i \sigma_{y}$ in $A$ is zero and the coefficient of $i \sigma_{x}$ in $A$ is positive. The restrictions on the ranges of $\beta$ and $r$ follow from the fact that, since $[\rho] \in P_{4}$, the matrices $A$ and $B$ do not commute. The uniqueness of the representative follows from the fact that the coefficients of $i \sigma_{x}$ in $A$ and $i \sigma_{z}$ in $B$ are nonzero.

Definition 2.4. Define maps $p_{1}: P_{4} \rightarrow S U(2) \times S U(2)$ and $p_{2}: P_{4} \rightarrow S^{2} \times S^{2}$ by

$$
p_{1}([\rho])=(A, B), \quad p_{2}([\rho])=(\hat{a},-\hat{b}),
$$

where $(A, B, a=i \hat{a} \cdot \vec{\sigma}, b=i \hat{b} \cdot \vec{\sigma})$ is the unique representative of $[\rho]$ such that $A$ and $B$ have the form given in equation (9).

We now want to consider the fiber $\mu^{-1}(t)$ over a fixed value $t \in[-1,1)$. Substituting equations $(9]$ into equation (8), we find that

$$
t=r^{2}+\left(1-r^{2}\right) \cos 2 \beta
$$

We solve equation (11) for $r$ to obtain

$$
r=\left(\frac{t-\cos 2 \beta}{1-\cos 2 \beta}\right)^{1 / 2}
$$

From equation (12), we see that for a fixed fixed value of $t \in[-1,1)$ the parameter $\beta$ must lie in the range $\left[\beta_{0}, \pi-\beta_{0}\right]$, where we have defined

$$
\beta_{0}:=(1 / 2) \cos ^{-1} t \in(0, \pi / 2] .
$$

Substituting equation $(12)$ into equation (9), we find that the unique representative of $[\rho]$ can be expressed as

$$
A=\left(\frac{t-\cos 2 \beta}{1-\cos 2 \beta}\right)^{1 / 2}\left(\cos \alpha+i \sigma_{z} \sin \alpha\right)+i \sigma_{x}\left(\frac{1-t}{1-\cos 2 \beta}\right)^{1 / 2}, \quad B=\cos \beta+i \sigma_{z} \sin \beta,
$$

where $\alpha \in[0,2 \pi], \beta \in\left[\beta_{0}, \pi-\beta_{0}\right], t \in[-1,1)$.

We will argue that the matrices $A$ and $B$ can be recovered from the value of $t \in[-1,1)$ and the matrices $a$ and $b$. This is clear for the case $t=-1$, for which the matrices $a$ and $b$ are not even needed:

Lemma 2.1. The space $p_{1}\left(\mu^{-1}(-1)\right)$ consists of the single point $\left(i \sigma_{x}, i \sigma_{z}\right)$.

Proof. Substituting $t=-1$ into equation (13), we find that $\beta_{0}=\pi / 2$. Since $\beta \in\left[\beta_{0}, \pi-\beta_{0}\right]$, the only allowed value of $\beta$ is $\beta=\pi / 2$. The result now follows from substituting $t=-1$ and $\beta=\pi / 2$ into equations (14).

Now consider the case $t \in(-1,1)$. From equation 13 it follows that $\beta_{0} \in(0, \pi / 2)$. Using equation (14), a straightforward calculation shows that

$$
[A, B]=t+i \sqrt{1-t^{2}} \hat{v}(\alpha, \beta) \cdot \vec{\sigma},
$$


where the unit vector $\hat{v}(\alpha, \beta) \in S^{2}$ is given by

$$
\hat{v}(\alpha, \beta)=\left(\sqrt{1-z(\beta)^{2}} \sin (\alpha+\beta), \sqrt{1-z(\beta)^{2}} \cos (\alpha+\beta), z(\beta)\right)
$$

and we have defined a diffeomorphism $z:\left[\beta_{0}, \pi-\beta_{0}\right] \rightarrow[-1,1]$ by

$$
z(\beta)=-\sqrt{\frac{1-t}{1+t}} \cot \beta .
$$

Lemma 2.2. For $t \in(-1,1)$ we can define $\operatorname{map} p_{1}\left(\mu^{-1}(t)\right) \rightarrow S^{2},(A, B) \mapsto \hat{v}(\alpha, \beta)$, where $\alpha \in[0,2 \pi]$ and $\beta \in\left[\beta_{0}, \pi-\beta_{0}\right]$ are chosen such that equations (14) for $A$ and $B$ are satisfied. This map is well-defined and is a homeomorphism.

Proof. Define a space

$$
X=\left\{(\alpha, \beta) \mid \alpha \in[0,2 \pi], \beta \in\left[\beta_{0}, \pi-\beta_{0}\right]\right\} / \sim,
$$

where the equivalence relation $\sim$ is defined such that

$$
\left(\alpha, \beta_{0}\right) \sim\left(\alpha^{\prime}, \beta_{0}\right), \quad\left(\alpha, \pi-\beta_{0}\right) \sim\left(\alpha^{\prime}, \pi-\beta_{0}\right), \quad(0, \beta) \sim(2 \pi, \beta) .
$$

We define a map $X \rightarrow p_{1}\left(\mu^{-1}(t)\right),[(\alpha, \beta)] \mapsto(A, B)$, where $A$ and $B$ are determined from $(\alpha, \beta, t)$ using equation (14). From equation (14), it is clear that this map is well-defined and is a homeomorphism. We define a map $X \rightarrow S^{2},[(\alpha, \beta)] \mapsto \hat{v}(\alpha, \beta)$, where $\hat{v}(\alpha, \beta)$ is given by equation (16). From equation (16), it is clear that this map is well-defined and is a homeomorphism.

Theorem 2.2. The space $P_{4}$ is homeomorphic to $S^{2} \times S^{2}-\Delta$, where $\Delta=\{(\hat{r}, \hat{r})\}$ is the diagonal. All representations in $P_{4}$ are nonabelian.

Proof. Consider the map $p_{2}: P_{4} \rightarrow S^{2} \times S^{2}$. Clearly the image of $p_{2}$ lies in $S^{2} \times S^{2}-\Delta$, since if $p_{2}([\rho]) \in \Delta$ then $b=a^{-1}$, which implies that $\mu([\rho])=(1 / 2) \operatorname{tr}\left((a b)^{-1}\right)=1$ and hence $[\rho] \notin P_{4}$. We can define an inverse map $S^{2} \times S^{2}-\Delta \rightarrow P_{4}$ as follows. Given a point $(\hat{a},-\hat{b}) \in S^{2} \times S^{2}-\Delta$, define $a=i \hat{a} \cdot \vec{\sigma}$ and $b=i \hat{b} \cdot \vec{\sigma}$. We have that

$$
a b=t+i \vec{v} \cdot \vec{\sigma},
$$

where we have defined

$$
t:=-\hat{a} \cdot \hat{b}, \quad \vec{v}:=-\hat{a} \times \hat{b} .
$$

If $t=-1$ then map $(\hat{a}, \hat{b})$ to $\left[\left(i \sigma_{x}, i \sigma_{z}, a, b\right)\right]$, otherwise map $(\hat{a}, \hat{b})$ to $[(A, B, a, b)]$, where $A$ and $B$ are determined from $t$ and $\hat{v}(\alpha, \beta):=-\vec{v} /|\vec{v}|$ via the homeomorphism $p_{1}\left(\mu^{-1}(t)\right) \rightarrow S^{2}$ defined in Lemma 2.2. By Lemmas 2.1 and 2.2, this inverse map is well-defined. The fact that all representations in $P_{4}$ are nonabelian is clear from the definition of the space $P_{4}$.

Remark 2.5. From a straightforward generalization of Lemma 2.2 it follows that $R\left(T^{2}, 1\right)$ is homeomorphic to $S^{2}$. This is consistent with the fact that, as noted in Remark 2.1 the space $R\left(T^{2}, 1\right)$ is homeomorphic to the moduli space of semistable parabolic bundles $M\left(T^{2}, 1\right)$, which is known to have the structure of an algebraic variety isomorphic to $\mathbb{C P}^{1}$ (see [4). We will not use the space $R\left(T^{2}, 1\right)$ here.

2.1.2. The piece $P_{3} \subset R\left(T^{2}, 2\right)$. We define the piece $P_{3} \subset R\left(T^{2}, 2\right)$ to be the set of conjugacy classes $[\rho] \in R\left(T^{2}, 2\right)$ such that $\mu([\rho])=1$. For any homomorphism $\rho: \pi_{1}\left(T^{2}-\left\{p_{1}, p_{2}\right\}\right) \rightarrow S U(2)$ representing a conjugacy class $[\rho] \in P_{3}$, the corresponding matrices $A$ and $B$ commute. We can therefore make the following definition:

Definition 2.5. We define a map $p: P_{3} \rightarrow R\left(T^{2}\right),[(A, B, a, b)] \mapsto[(A, B)]$.

Definition 2.6. We define a space $Y$ by

$$
Y=\left\{(\alpha, \beta, z)|\alpha \in[0,2 \pi], \beta \in[0, \pi],| z \mid \leq \sin ^{2} \alpha+\sin ^{2} \beta\right\} / \sim,
$$

where the equivalence relation $\sim$ is defined such that

$$
(\alpha, 0, z) \sim(2 \pi-\alpha, 0,-z)
$$$$
(\alpha, \pi, z) \sim(2 \pi-\alpha, \pi,-z),
$$$$
(0, \beta, z) \sim(2 \pi, \beta, z) .
$$

The space $Y$ is depicted in Figure 4 


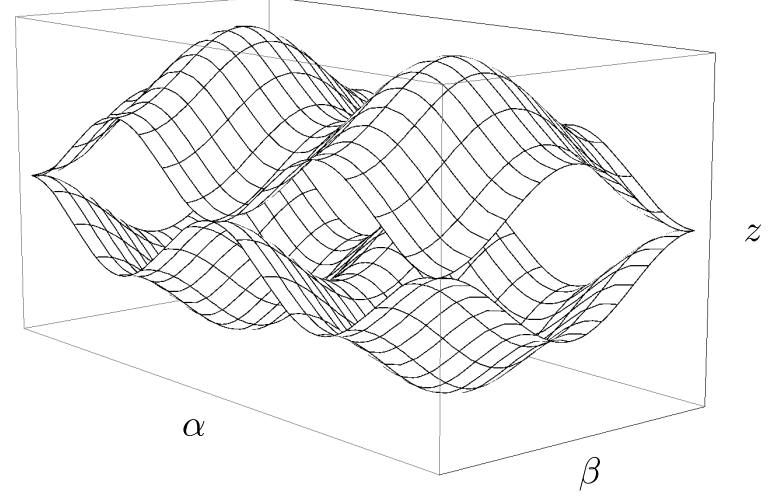

Figure 4. The space $Y$, which is homeomorphic to the piece $P_{3}$. The vertical faces are identified as described in Definition 2.6.

Theorem 2.3. The space $P_{3}$ is homeomorphic to $Y$. Abelian representations correspond to the boundary of $Y$ and nonabelian representations correspond to the interior of $Y$.

Proof. We first determine the fibers of the map $p: P_{3} \rightarrow R\left(T^{2}\right)$. Given a conjugacy class $[\rho] \in P_{3}$, we can always choose a representative of the form

$$
A=\cos \alpha+i \sigma_{z} \sin \alpha, \quad B=\cos \beta+i \sigma_{z} \sin \beta, \quad a=i \sigma_{x} \cos \gamma+i \sigma_{z} \sin \gamma, \quad b=a^{-1},
$$

where $\alpha \in[0,2 \pi], \beta \in[0, \pi]$, and $\gamma \in[-\pi / 2, \pi / 2]$. For $(A, B)=( \pm 1, \pm 1)$, we can conjugate so as to force $\gamma=0$. From these considerations it follows that the fibers of $p$ are points $(\gamma=0)$ over the four pillowcase points $[(A, B)]=[( \pm 1, \pm 1)]$, and intervals $(\gamma \in[-\pi / 2, \pi / 2])$ over all other points. We can thus define a homeomorphism $P_{3} \rightarrow Y$ by

$$
(\alpha, \beta, \gamma) \mapsto\left(\alpha, \beta,(2 \gamma / \pi)\left(\sin ^{2} \alpha+\sin ^{2} \beta\right)\right),
$$

where $\alpha \in[0,2 \pi], \beta \in[0, \pi]$, and $\gamma \in[-\pi / 2, \pi / 2]$ are chosen such that equations 24 are satisfied. From equations (24), it is clear that under this homeomorphism abelian representations correspond to the boundary of $Y$ and nonabelian representations correspond to the interior of $Y$.

Remark 2.6. In what follows we will use $(\alpha, \beta, \gamma)$ as coordinates on $P_{3}$, subject to the identifications

$$
(\alpha, \beta, \gamma) \sim(\alpha+2 \pi, \beta, \gamma), \quad(\alpha, \beta, \gamma) \sim(\alpha, \beta+2 \pi, \gamma), \quad(\alpha, \beta, \gamma) \sim(-\alpha,-\beta,-\gamma),
$$

and if $(\alpha, \beta) \in\{(0,0),(0, \pi),(\pi, 0),(\pi, \pi)\}$, corresponding to the four pillowcase points of $R\left(T^{2}\right)$, then $(\alpha, \beta, \gamma) \sim(\alpha, \beta, 0)$.

Remark 2.7. Note that $P_{3}$ deformation retracts onto $P_{3} \cap\{\gamma=0\}$, which may be identified with the pillowcase $R\left(T^{2}\right)$.

Remark 2.8. Theorems 2.2 and 2.3 imply Theorem 1.1 from the introduction.

Remark 2.9. As noted in Remark 2.1 the space $R\left(T^{2}, 2\right)$ is homeomorphic to the moduli space of semistable parabolic bundles $M\left(T^{2}, 2\right)$, which is known to have the the structure of an algebraic variety isomorphic to $\mathbb{C P}^{1} \times \mathbb{C P}^{1}$ (see [4, 20]). It follows that $R\left(T^{2}, 2\right)$ is homeomorphic to $S^{2} \times S^{2}$, although this does not seem to be easy to show from our description of this space.

2.2. The character variety $R\left(S^{1} \times D^{2}, A_{1}\right)$. Our next task is to determine the Lagrangian in $R\left(T^{2}, 2\right)$ that corresponds to a solid torus containing an unknotted arc. Consider a solid torus $S^{1} \times D^{2}$ containing an unknotted $\operatorname{arc} A_{1}$ connecting points $p_{1}$ and $p_{2}$ on the boundary.

Definition 2.7. We define the character variety $R\left(S^{1} \times D^{2}, A_{1}\right)$ to be the space of conjugacy classes of homomorphisms $\rho: \pi_{1}\left(S^{1} \times D^{2}-A_{1}\right) \rightarrow S U(2)$ that map loops around the arc $A_{1}$ to traceless matrices. 
Theorem 2.4. The space $R\left(S^{1} \times D^{2}, A_{1}\right)$ is homeomorphic to the closed unit disk $D^{2}$. A specific homeomorphism is given by $D^{2} \rightarrow R\left(S^{1} \times D^{2}, A_{1}\right),(x, y) \mapsto[\rho]$, where $\rho$ is specified by $S U(2)$-matrices of the form

$$
\begin{aligned}
a & =i \sigma_{z}, \\
b & =a^{-1} \\
A & =x+i\left(1-x^{2}-y^{2}\right)^{1 / 2} \sigma_{x}+i y \sigma_{z}, \\
B & =1 .
\end{aligned}
$$

Abelian representations correspond to the boundary of $D^{2}$, for which $x^{2}+y^{2}=1$, and nonabelian representations correspond to the interior of $D^{2}$, for which $x^{2}+y^{2}<1$.

Proof. The fundamental group of $S^{1} \times D^{2}-A_{1}$ is given by

$$
\pi_{1}\left(S^{1} \times D^{2}-A_{1}\right)=\left\langle A, B, a, b \mid B=1, b=a^{-1}\right\rangle,
$$

where $A$ and $B$ are the longitude and meridian of the boundary of the solid torus and $a$ and $b$ are loops in the boundary encircling the points $p_{1}$ and $p_{2}$, respectively.

We now consider homomorphisms $\rho: \pi_{1}\left(S^{1} \times D^{2}-A_{1}\right) \rightarrow S U(2)$ that satisfy the requirements described in Definition 2.7 for $R\left(S^{1} \times D^{2}, A_{1}\right)$. As usual, we use the same notation for generators of the fundamental group and their images under $\rho$; for example, we denote $\rho(A)$ by $A$. Given an arbitrary representative $\rho$ of a conjugacy class $[\rho] \in R\left(S^{1} \times D^{2}, A_{1}\right)$, we will argue that we can always conjugate so as to obtain a unique representative of the form given in equations (27)- (30). We first conjugate so that $a=b^{-1}=i \sigma_{z}$. We then rotate about the $z$-axis so that the coefficient of $i \sigma_{y}$ in $A$ is zero and the coefficient of $i \sigma_{x}$ in $A$ is nonnegative. We have thus obtained a representative of the form given in equations (27)-(30), and it is clear from these equations that such a representative is unique.

Remark 2.10. It will be useful to consider an alternative parameterization of $R\left(S^{1} \times D^{2}, A_{1}\right)$. Given $(x, y) \in D^{2}$, define angles $\chi$ and $\psi$ by

$$
\chi=\cos ^{-1} x \in[0, \pi], \quad \psi=\sin ^{-1}\left(\frac{y}{\sqrt{1-x^{2}}}\right) \in[-\pi / 2, \pi / 2],
$$

with the convention that $\psi=0$ for $(x= \pm 1, y=0)$. Then equation 29 for $A$ becomes

$$
A=\cos \chi+i \sin \chi\left(\sigma_{x} \cos \psi+\sigma_{z} \sin \psi\right) \text {. }
$$

Now conjugate equations 27 -30$)$ by $g=\left(i \sigma_{z}\right) e^{i(\pi / 4-\psi / 2) \sigma_{y}}$ to obtain a representative such that

$$
\begin{aligned}
& a=i \sigma_{x} \cos \psi+i \sigma_{z} \sin \psi, \\
& b=a^{-1}, \\
& A=\cos \chi+i \sigma_{z} \sin \chi=e^{i \chi \sigma_{z},} \\
& B=1 .
\end{aligned}
$$

Equations (34)-(37) with $\chi \in[0, \pi]$ and $\psi \in[-\pi / 2, \pi / 2]$ constitute an alternative parameterization of $R\left(S^{1} \times D^{2}, A_{1}\right)$ that is better suited for calculations.

Given a representation of $\pi_{1}\left(S^{1} \times D^{2}-A_{1}\right)$, we can pull back along the inclusion $T^{2}-\left\{p_{1}, p_{2}\right\} \rightarrow$ $S^{1} \times D^{2}-A_{1}$ to obtain a representation of $\pi_{1}\left(T^{2}-\left\{p_{1}, p_{2}\right\}\right)$. This induces a map $R\left(S^{1} \times D^{2}, A_{1}\right) \rightarrow R\left(T^{2}, 2\right)$.

Definition 2.8. We will refer to the image of $R\left(S^{1} \times D^{2}, A_{1}\right)$ in $R\left(T^{2}, 2\right)$ as the disk Lagrangian $L_{d}$, and we will denote the image in $R\left(T^{2}, 2\right)$ of the point in $R\left(S^{1} \times D^{2}, A_{1}\right)$ with coordinates $(\chi, \psi)$ by $L_{d}(\chi, \psi)$.

Theorem 2.5. The map $R\left(S^{1} \times D^{2}, A_{1}\right) \rightarrow R\left(T^{2}, 2\right)$ is an injection whose image lies in the piece $P_{3}$. In terms of the parameterization $(\chi, \psi)$ of $R\left(S^{1} \times D^{2}, A_{1}\right)$ described in Remark 2.10, we have that

$$
\alpha\left(L_{d}(\chi, \psi)\right)=\chi, \quad \beta\left(L_{d}(\chi, \psi)\right)=0, \quad \gamma\left(L_{d}(\chi, \psi)\right)=\psi .
$$

Proof. This follows directly from equations $(34)-(37)$ and the definition of the coordinates $(\alpha, \beta, \gamma)$ in equation (24). It is clear from equations (38) that the map is injective.

Remark 2.11. Theorems 2.4 and 2.5 imply Theorem 1.2 from the introduction. 

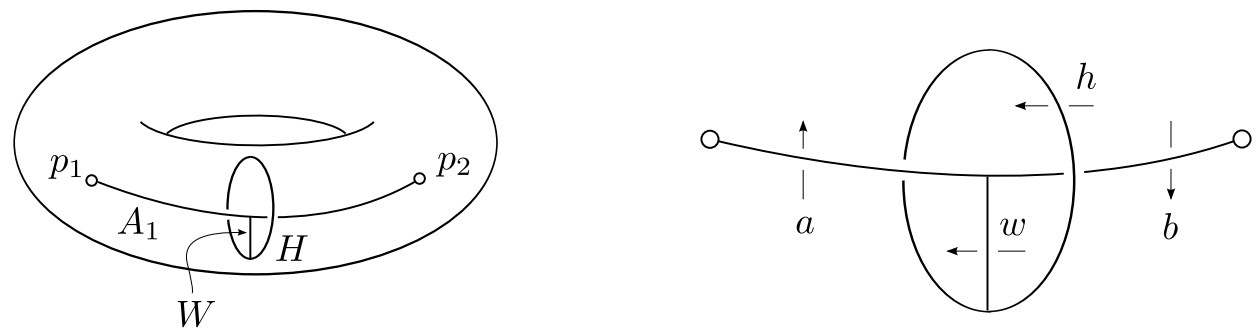

Figure 5. (Left) Solid torus $S^{1} \times D^{2}-A_{1}$ used to define $R^{\natural}\left(S^{1} \times D^{2}, A_{1}\right)$. Shown are the $\operatorname{arc} A_{1}$, the loop $H$, and the arc $W$. (Right) Loops $a, b, h$, and $w$.

2.3. The character variety $R^{\natural}\left(S^{1} \times D^{2}, A_{1}\right)$. In order to avoid reducible connections, it is necessary to modify the character variety considered in the previous section. Specifically, as shown in Figure 5, we modify the solid torus containing the unknotted arc $A_{1}$ by introducing a small loop $H$ around the arc, together with an $\operatorname{arc} W$ connecting the $\operatorname{arc} A_{1}$ to the loop $H$.

Definition 2.9. We define the character variety $R^{\natural}\left(S^{1} \times D^{2}, A_{1}\right)$ to be the space of conjugacy classes of homomorphisms $\rho: \pi_{1}\left(S^{1} \times D^{2}-A_{1} \cup H \cup W\right) \rightarrow S U(2)$ that maps loops around $A_{1}$ and $H$ to traceless matrices and loops around $W$ to -1 .

Theorem 2.6. The space $R^{\natural}\left(S^{1} \times D^{2}, A_{1}\right)$ is homeomorphic to $S^{3}$. A specific homeomorphism $S^{3} \rightarrow$ $R^{\natural}\left(S^{1} \times D^{2}, A_{1}\right)$ is given by $\left(z_{1}, z_{2}\right) \mapsto[\rho]$, where $S^{3}=\left\{\left.\left(z_{1}, z_{2}\right) \in \mathbb{C}^{2}|| z_{1}\right|^{2}+\left|z_{2}\right|^{2}=1\right\}$ and $\rho$ is specified by $S U(2)$-matrices of the form

$$
\begin{aligned}
a & =i \sigma_{z}, \\
b & =a^{-1}, \\
A & =\left(\begin{array}{cc}
z_{1} & -\bar{z}_{2} \\
z_{2} & \bar{z}_{1}
\end{array}\right), \\
B & =1, \\
h & =i \sigma_{x}, \\
w & =-1 .
\end{aligned}
$$

All representations in $R^{\natural}\left(S^{1} \times D^{2}, A_{1}\right)$ are nonabelian.

Proof. We define homotopy classes of loops $A, B, a, b, h$, and $w$ as shown in Figure 5 , and read off relations from Figure 5 to obtain a presentation of $\pi_{1}\left(S^{1} \times D^{2}-A_{1} \cup H \cup W\right)$ :

$$
\pi_{1}\left(S^{1} \times D^{2}-A_{1} \cup H \cup W\right)=\left\langle A, B, a, b, h, w \mid h w a=a h, b=a^{-1}, B=1\right\rangle .
$$

We now consider homomorphisms $\rho: \pi_{1}\left(S^{1} \times D^{2}-A_{1} \cup H \cup W\right) \rightarrow S U(2)$ that satisfy the requirements described in Definition 2.9 for $R^{\natural}\left(S^{1} \times D^{2}, A_{1}\right)$. As usual, we use the same notation for generators of the fundamental group and their images under $\rho$; for example, we denote $\rho(A)$ by $A$. Given an arbitrary representative $\rho$ of a conjugacy class $[\rho] \in R^{\natural}\left(S^{1} \times D^{2}, A_{1}\right)$, will argue that we can always conjugate so as to obtain a unique representative of the form given in equations (39)-(44). We first conjugate so that $a=b^{-1}=i \sigma_{z}$. Since $w=-1$, the relation $h w a=a h$ implies that $a$ and $h$ anticommute, so $h$ must have the form $h=i \sigma_{x} \cos \theta+i \sigma_{y} \sin \theta$ for some angle $\theta$. Now rotate about the $z$-axis so that $h=i \sigma_{x}$. We have thus obtained a representative of the form given in equations (39)-(44). From the fact that $a=i \sigma_{z}$ and $h=i \sigma_{x}$ it follows that such a representative is unique.

Given a representation of $\pi_{1}\left(S^{1} \times D^{2}-A_{1} \cup H \cup W\right)$, we can pull back along the inclusion $S^{1} \times D^{2}-$ $A_{1} \cup H \cup W \rightarrow S^{1} \times D^{2}-A_{1}$ to obtain a representation of $\pi_{1}\left(S^{1} \times D^{2}-A_{1}\right)$. This induces a map $R^{\natural}\left(S^{1} \times D^{2}, A_{1}\right) \rightarrow R\left(S^{1} \times D^{2}, A_{1}\right)$.

Theorem 2.7. The pullback map $R^{\natural}\left(S^{1} \times D^{2}, A_{1}\right) \rightarrow R\left(S^{1} \times D^{2}, A_{1}\right)$ is given by

$$
x+i y=z_{1} .
$$



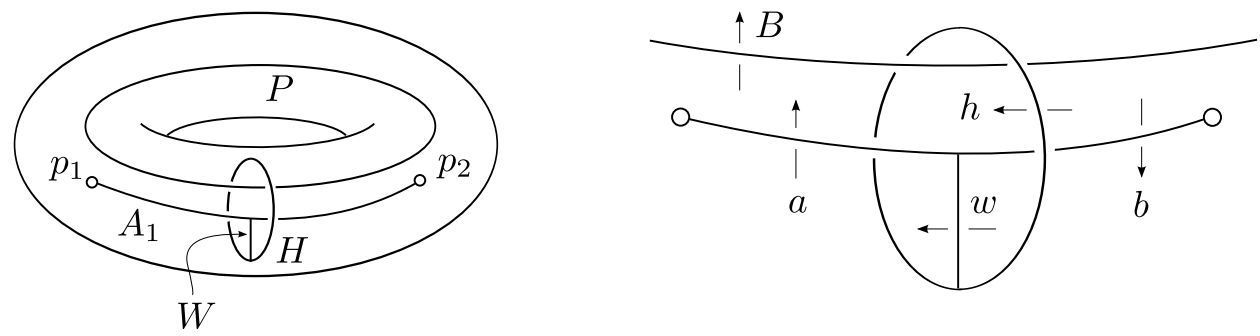

Figure 6. (Left) Solid torus $S^{1} \times D^{2}-A_{1}$ used to define $R_{\pi}^{\natural}\left(S^{1} \times D^{2}, A_{1}\right)$. Shown are the $\operatorname{arc} A_{1}$, the loop $H$ and $\operatorname{arc} W$, and the perturbation loop $P$. (Right) Loops $B, a, b, h$, and $w$.

This map is surjective. The fibers of the map are points for abelian representations and circles for nonabelian representations.

Proof. The fact that the map is given by $x+i y=z_{1}$ follows from conjugating equations $(39)-(42)$ so that they have the form given in equations (27)-30). The surjectivity of the map is clear. The fiber over the point $(x, y)$ is parameterized by

$$
\left\{\left.\left(z_{1}, z_{2}\right)\left|z_{1}=x+i y,\right| z_{1}\right|^{2}+\left|z_{2}\right|^{2}=1\right\} .
$$

This set is the singleton $\left\{\left(z_{1}, 0\right)\right\}$ if $\left|z_{1}\right|^{2}=x^{2}+y^{2}=1$, corresponding to an abelian representation, and a circle if $\left|z_{1}\right|^{2}=x^{2}+y^{2}<1$, corresponding to a nonabelian representation.

2.4. The character variety $R_{\pi}^{\natural}\left(S^{1} \times D^{2}, A_{1}\right)$. In order to render the Chern-Simons functional Morse, we must add a suitable holonomy perturbation term [15, 16]. The perturbation term vanishes outside of a solid torus, which we will assume lies entirely inside one of the solid tori in the Heegaard splitting of the lens space. We begin with the solid torus $S^{1} \times D^{2}$ considered in Section 2.3 , which contains the arc $A_{1}$, the loop $H$ encircling $A_{1}$, and the arc $W$ connecting $A_{1}$ and $H$. We now add an additional loop $P$, as shown in Figure 6. If we slightly thicken the loop $P$ we obtain the solid torus in which the holonomy perturbation term is nonzero. The net effect of the perturbation term is to impose an additional requirement on the homomorphisms $\rho: \pi_{1}\left(S^{1} \times D^{2}-A_{1} \cup H \cup W\right) \rightarrow S U(2)$. Specifically, letting $\lambda_{P}=h^{-1} A$ and $\mu_{P}=B$ denote the homotopy classes of the longitude and meridian of the solid torus obtained by thickening $P$, we require that if $\rho\left(\lambda_{P}\right)$ has the form

$$
\rho\left(\lambda_{P}\right)=e^{i \phi \hat{r} \cdot \sigma}=\cos \phi+i \hat{r} \cdot \vec{\sigma} \sin \phi
$$

for some angle $\phi$ and some unit vector $\hat{r} \in S^{2}$, then $\rho\left(\mu_{P}\right)$ must have the form

$$
\rho\left(\mu_{P}\right)=e^{i \nu \hat{r} \cdot \sigma}=\cos \nu+i \hat{r} \cdot \vec{\sigma} \sin \nu,
$$

where $\nu=\epsilon f(\phi)$. Here $\epsilon>0$ is a small parameter that controls the magnitude of the perturbation and $f: \mathbb{R} \rightarrow \mathbb{R}$ is a function such that $f(-x)=-f(x), f$ is $2 \pi$-periodic, and $f(x)$ is zero if and only if $x$ is a multiple of $\pi$. We will usually take $f(\phi)=\sin \phi$.

Definition 2.10. We define the character variety $R_{\pi}^{\natural}\left(S^{1} \times D^{2}, A_{1}\right)$ to be the space of conjugacy classes of homomorphisms $\rho: \pi_{1}\left(S^{1} \times D^{2}-A_{1} \cup H \cup W \cup P\right) \rightarrow S U(2)$ that take loops around $A_{1}$ and $H$ to traceless matrices and loops around $W$ to -1 , and are such that if $\rho\left(\lambda_{P}\right)$ has the form given in equation (48) then $\rho\left(\lambda_{P}\right)$ must have the form given in equation 49 .

Theorem 2.8. For $\epsilon>0$ sufficiently small, the space $R_{\pi}^{\natural}\left(S^{1} \times D^{2}, A_{1}\right)$ is homeomorphic to $S^{2}$. A specific homeomorphism $S^{2} \rightarrow R_{\pi}^{\natural}\left(S^{1} \times D^{2}, A_{1}\right)$ is given by $(\phi, \theta) \rightarrow[\rho]$, where $\phi \in[0, \pi]$ and $\theta \in[0,2 \pi]$ are 
spherical-polar coordinates on $S^{2}$ and $\rho$ is specified by $S U(2)$-matrices of the form

$$
\begin{aligned}
a & =i \sigma_{z}, \\
b & =-h a^{-1} h^{-1}=-\left(\cos ^{2} \nu+\sin ^{2} \nu \sin ^{2} \theta\right)^{-1}\left(i \sigma_{x} \sin 2 \nu \sin \theta+i \sigma_{z}\left(\cos ^{2} \nu-\sin ^{2} \nu \sin ^{2} \theta\right)\right), \\
A & =h\left(\cos \phi+i \sin \phi\left(\sigma_{x} \cos \theta+\sigma_{y} \sin \theta\right),\right. \\
B & =\cos \nu+i \sin \nu\left(\sigma_{x} \cos \theta+\sigma_{y} \sin \theta\right), \\
h & =\left(\cos ^{2} \nu+\sin ^{2} \nu \sin ^{2} \theta\right)^{-1 / 2}\left(i \sigma_{x} \cos \nu-i \sigma_{z} \sin \nu \sin \theta\right), \\
w & =-1,
\end{aligned}
$$

and $\nu=\epsilon \sin \phi$.

Proof. We define homotopy classes of loops $A, B, a, b$, and $h$ as shown in Figure 6 , and read off relations from Figure 6 to obtain a presentation of $\pi_{1}\left(S^{1} \times D^{2}-A_{1} \cup H \cup W \cup P\right)$ :

$$
\pi_{1}\left(S^{1} \times D^{2}-A_{1} \cup H \cup W \cup P\right)=\left\langle A, B, a, b, h, w \mid h w a B=a B h, b=h a^{-1} w^{-1} h^{-1}\right\rangle
$$

We now consider homomorphisms $\rho: \pi_{1}\left(S^{1} \times D^{2}-A_{1} \cup H \cup W \cup P\right) \rightarrow S U(2)$ that satisfy the requirements described in Definition 2.10 for $R_{\pi}^{\natural}\left(S^{1} \times D^{2}, A_{1}\right)$. As usual, we use the same notation for generators of the fundamental group and their images under $\rho$; for example, we denote $\rho(A)$ by $A$. Given an arbitrary representative $\rho$ of a conjugacy class $[\rho] \in R_{\pi}^{\natural}\left(S^{1} \times D^{2}, A_{1}\right)$, we will argue that we can always conjugate so as to obtain a unique representative of the form given in equations (50)-(55). We first conjugate so that $a=i \sigma_{z}$. Next, we rotate about the $z$-axis so that the coefficient of $i \sigma_{y}$ in $h$ is zero. After these operations have been performed, we express $\lambda_{P}$ in the form

$$
\lambda_{P}=\cos \phi+i \hat{r} \cdot \vec{\sigma} \sin \phi
$$

for some angle $\phi$ and some unit vector $\hat{r} \in S^{2}$. The relationship between $\lambda_{P}$ and $\mu_{P}$ described in equations (48) and 49 then implies that

$$
B=\mu_{P}=\cos \nu+i \hat{r} \cdot \vec{\sigma} \sin \nu
$$

where $\nu=\epsilon \sin \phi$. We also find that

$$
A=h \lambda_{P}=h(\cos \phi+i \hat{r} \cdot \vec{\sigma} \sin \phi) .
$$

Since $w=-1$, the relation $b=h a^{-1} w^{-1} h^{-1}$ implies that $b=-h a^{-1} h^{-1}$, and the relation $h w a B=a B h$ implies that $a B$ and $h$ anticommute. The fact that $a B$ and $h$ anticommute implies that the $z$-component of $\hat{r}$ must vanish, so $\hat{r}=(\cos \theta, \sin \theta, 0)$ for some angle $\theta$. The fact that $a B$ and $h$ anticommute, in conjunction with the fact that the coefficient of $i \sigma_{y}$ in $h$ is zero, further implies that $h$ must have the form

$$
h= \pm\left(\cos ^{2} \nu+\sin ^{2} \nu \sin ^{2} \theta\right)^{-1 / 2}\left(i \sigma_{x} \cos \nu-i \sigma_{z} \sin \nu \sin \theta\right) .
$$

In fact, we can assume that the plus sign obtains in equation 60 , since if not then we can conjugate by $i \sigma_{z}$ and redefine $\theta \mapsto \theta+\pi$; this operation flips the signs of $h$ and $A$ and leaves $B, a, b$, and $w$ invariant. We have thus obtained a representative of the form given in equations (50)-(55). Since $a=i \sigma_{z}$ and the coefficient of $i \sigma_{x}$ in $h$ is nonzero, such a representative is unique.

We note that equations (50)-(54) are invariant under the transformations

$$
(\phi, \theta) \mapsto(\phi+2 \pi, \theta), \quad(\phi, \theta) \mapsto(\phi, \theta+2 \pi), \quad(\phi, \theta) \mapsto(-\phi, \theta+\pi) .
$$

By invariance under the second transformation we can assume that $\phi \in[-\pi, \pi]$, by invariance under the third transformation we can further assume that $\phi \in[0, \pi]$, and by invariance under the first transformation we can assume that $\theta \in[0,2 \pi]$. From equations $(50)-(55)$, it is clear that the map $S^{2} \rightarrow R_{\pi}^{\natural}\left(S^{1} \times D^{2}, A_{1}\right)$, $(\phi, \theta) \mapsto[\rho]$ is a homeomorphism, where $(\phi, \theta)$ are spherical-polar coordinates on $S^{2}$.

Remark 2.12. It is useful to define Cartesian coordinates $(x, y, z)$ on $R_{\pi}^{\natural}\left(S^{1} \times D^{2}, A_{1}\right)$ by

$$
(x, y, z)=(\sin \phi \sin \theta, \sin \phi \sin \theta, \cos \phi) .
$$

Given a representation of $\pi_{1}\left(S^{1} \times D^{2}-A_{1} \cup H \cup W \cup P\right)$, we can pull back along the inclusion $S^{1} \times$ $D^{2}-A_{1} \cup H \cup W \cup P \rightarrow T^{2}-\left\{p_{1}, p_{2}\right\}$ to obtain a representation of $\pi_{1}\left(T^{2}-\left\{p_{1}, p_{2}\right\}\right)$. This induces a map $R_{\pi}^{\natural}\left(S^{1} \times D^{2}, A_{1}\right) \rightarrow R\left(T^{2}, 2\right)$. 
Definition 2.11. We will say that the image of $R_{\pi}^{\natural}\left(S^{1} \times D^{2}, A_{1}\right)$ in $R\left(T^{2}, 2\right)$ is the sphere Lagrangian $L_{s}$, and we will denote the image in $R\left(T^{2}, 2\right)$ of the point in $R_{\pi}^{\natural}\left(S^{1} \times D^{2}, A_{1}\right)$ with coordinates $(\phi, \theta)$ by $L_{s}(\phi, \theta)$.

Remark 2.13. As noted in Remark 2.2 for each element $\lambda \in \pi_{1}\left(T^{2}-\left\{p_{1}, p_{2}\right\}\right)$ there is is a corresponding function $R\left(T^{2}, 2\right) \rightarrow \mathbb{R}$ given by $[\rho] \mapsto \operatorname{tr}(\rho(\lambda))$. In performing calculations it will be useful to evaluate certain functions of this form at the point $L_{s}(\phi, \theta) \in R\left(T^{2}, 2\right)$. Using equations $(50)-(53)$, we find that

$$
\begin{aligned}
(\operatorname{tr} A)\left(L_{s}(\phi, \theta)\right) & =-\frac{2 \cos \nu \cos \theta \sin \phi}{\sqrt{\cos ^{2} \nu+\sin ^{2} \nu \sin ^{2} \theta}}, \\
(\operatorname{tr} B)\left(L_{s}(\phi, \theta)\right) & =2 \cos \nu, \\
(\operatorname{tr} A a)\left(L_{s}(\phi, \theta)\right) & =\frac{2 \sin (\phi+\nu) \sin \theta}{\sqrt{\cos ^{2} \nu+\sin ^{2} \nu \sin ^{2} \theta}}, \\
(\operatorname{tr} B a)\left(L_{s}(\phi, \theta)\right) & =0, \\
(\operatorname{tr} A b)\left(L_{s}(\phi, \theta)\right) & =-\frac{2 \sin (\phi-\nu) \sin \theta}{\sqrt{\cos ^{2} \nu+\sin ^{2} \nu \sin ^{2} \theta}}, \\
(\operatorname{tr} A B)\left(L_{s}(\phi, \theta)\right) & =-\frac{2 \cos \nu \cos \theta \sin (\phi+\nu)}{\sqrt{\cos ^{2} \nu+\sin ^{2} \nu \sin ^{2} \theta}}, \\
\mu\left(L_{s}(\phi, \theta)\right) & =\frac{\cos ^{2} \nu-\sin ^{2} \nu \sin ^{2} \theta}{\cos ^{2} \nu+\sin ^{2} \nu \sin ^{2} \theta} .
\end{aligned}
$$

Theorem 2.9. The map $R_{\pi}^{\natural}\left(S^{1} \times D^{2}, A_{1}\right) \cap\{y \neq 0\} \rightarrow R\left(T^{2}, 2\right)$ is an injection whose image lies in $P_{4}$. In terms of the parameterization $(\phi, \theta)$ of $R_{\pi}^{\natural}\left(S^{1} \times D^{2}, A_{1}\right)$ given in Theorem 2.8, we have that

$$
\begin{aligned}
\hat{a}\left(L_{s}(\phi, \theta)\right) & =(-\sin (\phi+\nu),-\cos (\phi+\nu), 0), \\
\hat{b}_{x}\left(L_{s}(\phi, \theta)\right) & =\left(\cos ^{2} \nu+\sin ^{2} \nu \sin ^{2} \theta\right)^{-1}\left(\cos ^{2} \nu \cos ^{2} \theta \sin (\phi+\nu)+\sin ^{2} \theta \sin (\phi-\nu)\right), \\
\hat{b}_{y}\left(L_{s}(\phi, \theta)\right) & =\left(\cos ^{2} \nu+\sin ^{2} \nu \sin ^{2} \theta\right)^{-1}\left(\cos ^{2} \nu \cos ^{2} \theta \cos (\phi+\nu)+\sin ^{2} \theta \cos (\phi-\nu)\right), \\
\hat{b}_{z}\left(L_{s}(\phi, \theta)\right) & =-(1 / 2)\left(\cos ^{2} \nu+\sin ^{2} \nu \sin ^{2} \theta\right)^{-1} \sin (2 \nu) \sin (2 \theta)
\end{aligned}
$$

for $\theta \in(0, \pi)$, and

$$
\begin{aligned}
\hat{a}\left(L_{s}(\phi, \theta)\right) & =(\sin (\phi+\nu), \cos (\phi+\nu), 0), \\
\hat{b}_{x}\left(L_{s}(\phi, \theta)\right) & =-\left(\cos ^{2} \nu+\sin ^{2} \nu \sin ^{2} \theta\right)^{-1}\left(\cos ^{2} \nu \cos ^{2} \theta \sin (\phi+\nu)+\sin ^{2} \theta \sin (\phi-\nu)\right), \\
\hat{b}_{y}\left(L_{s}(\phi, \theta)\right) & =-\left(\cos ^{2} \nu+\sin ^{2} \nu \sin ^{2} \theta\right)^{-1}\left(\cos \nu^{2} \cos ^{2} \theta \cos (\phi+\nu)+\sin ^{2} \theta \cos (\phi-\nu)\right), \\
\hat{b}_{z}\left(L_{s}(\phi, \theta)\right) & =-(1 / 2)\left(\cos ^{2} \nu+\sin ^{2} \nu \sin ^{2} \theta\right)^{-1} \sin (2 \nu) \sin (2 \theta)
\end{aligned}
$$

for $\theta \in(\pi, 2 \pi)$.

Proof. To obtain equations $(70-\sqrt{77})$, we conjugate equations $50-53$ so that they have the form of equations $(9)$, then read off $\hat{a}$ and $\vec{b}$ from $a=i \hat{a} \cdot \vec{\sigma}$ and $b=i \hat{b} \cdot \vec{\sigma}$.

We will now show injectivity. Rather than work directly with equations $(70)-(77)$, which are rather complicated, we will argue that the coordinates $(\phi, \theta)$ can be determined from functions on $R\left(T^{2}, 2\right)$ of the form described in Remark 2.2. First we determine the coordinate $\theta$. From equations (64), 665), and (68), it follows that

$$
\theta=\operatorname{Arg}\left(-(\operatorname{tr} A B)\left(L_{s}(\phi, \theta)\right)+(i / 2)(\operatorname{tr} B)\left(L_{s}(\phi, \theta)\right)(\operatorname{tr} A a)\left(L_{s}(\phi, \theta)\right)\right) .
$$

Next we determine the coordinate $\phi$. Define a function $f: R\left(T^{2}, 2\right) \cap\{\operatorname{tr} A b \neq 0\} \rightarrow \mathbb{R}$ by

$$
f=-\frac{\operatorname{tr}(A a)}{\operatorname{tr}(A b)} \text {. }
$$

From equations (65) and (67) it follows that

$$
f\left(L_{s}(\phi, \theta)\right)=\frac{\sin (\phi+\nu)}{\sin (\phi-\nu)}=\frac{\sin (\phi+\epsilon \sin \phi)}{\sin (\phi-\epsilon \sin \phi)} .
$$




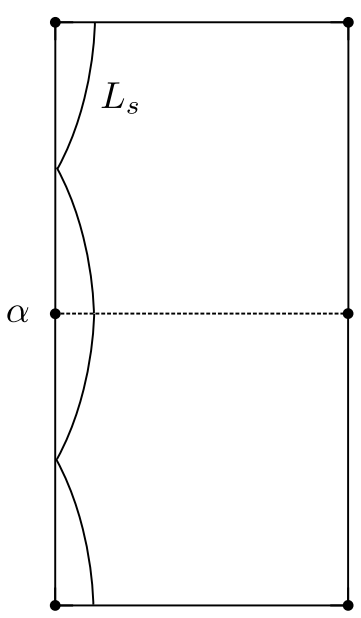

$\beta$

Figure 7. The sphere Lagrangian $L_{s}$ in the pillowcase $P_{3} \cap\{\gamma=0\}$.

Since $f\left(L_{s}(\phi, \theta)\right)$ is independent of $\theta$, we can define a function $F:(0, \pi) \rightarrow \mathbb{R}, F(\phi)=f\left(L_{s}(\phi, \theta)\right)$. It is straightforward to show that if $\epsilon$ is sufficiently small then $F^{\prime}(\phi)>0$ for all $\phi \in(0, \pi)$, hence $F$ is a diffeomorphism onto its image. We conclude that $f\left(L_{s}(\phi, \theta)\right)$ uniquely determines $\phi$.

Theorem 2.10. The image of the map $R_{\pi}^{\natural}\left(S^{1} \times D^{2}, A_{1}\right) \cap\{y=0\} \rightarrow R\left(T^{2}, 2\right)$ lies in the pillowcase $P_{3} \cap\{\gamma=0\}$. In terms of the parameterization $(\phi, \theta)$ of $R_{\pi}^{\natural}\left(S^{1} \times D^{2}, A_{1}\right)$ given in Theorem 2.8, we have that

$$
\begin{array}{llll}
\theta=0: & \alpha\left(L_{s}(\phi, \theta)\right)=\phi+\pi / 2, & \beta\left(L_{s}(\phi, \theta)\right)=\nu=\epsilon \sin \phi, & \gamma\left(L_{s}(\phi, \theta)\right)=0, \\
\theta=\pi: & \alpha\left(L_{s}(\phi, \theta)\right)=\phi-\pi / 2, & \beta\left(L_{s}(\phi, \theta)\right)=\nu=\epsilon \sin \phi, & \gamma\left(L_{s}(\phi, \theta)\right)=0 .
\end{array}
$$

The north pole $(\phi=0)$ and south pole $(\phi=\pi)$ both get mapped to the same point $(\alpha, \beta, \gamma)=(\pi / 2,0,0)$, and the map is injective elsewhere. The image of the map is depicted in Figure 7.

Proof. We substitute $\theta=0$ and $\theta=\pi$ into equations (50)-(53), then conjugate these equations into the form given by equations (24) and read off the coordinates $(\alpha, \beta, \gamma)$. The statement regarding the injectivity of the map is clear from these expressions.

Remark 2.14. Theorems 2.8, 2.9, and 2.10 imply Theorem 1.3 from the introduction.

\section{The Group $\mathrm{MCG}_{2}\left(T^{2}\right)$ AND ITS ACTION ON $R\left(T^{2}, 2\right)$}

\subsection{The mapping class group $\mathrm{MCG}_{2}\left(T^{2}\right)$ for the twice-punctured torus.}

Definition 3.1. Given a surface $S$ and $n$ distinct marked points $p_{1}, \cdots, p_{n} \in S$, we define the mapping class group $\mathrm{MCG}_{n}(S)$ to be the group of isotopy classes of orientation-preserving homeomorphisms of $S$ that fix $\left\{p_{1}, \cdots, p_{n}\right\}$ as a set, and we define the pure mapping class group $\operatorname{PMCG}_{n}(S)$ to be the subgroup of $\operatorname{MCG}_{n}(S)$ consisting of isotopy classes of orientation-preserving homeomorphisms of $S$ that fix each individual marked point $p_{1}, \cdots, p_{n}$.

There is a short exact sequence

$$
1 \longrightarrow \mathrm{PMCG}_{n}(S) \longrightarrow \mathrm{MCG}_{n}(S) \longrightarrow \Sigma_{n} \longrightarrow 1
$$

where $\Sigma_{n}$ is the symmetric group of permutations of $\left\{p_{1}, \cdots, p_{n}\right\}$ and the homomorphism $\mathrm{MCG}_{n}(S) \rightarrow \Sigma_{n}$ takes an isotopy class of homeomorphisms of $S$ to its corresponding permutation of marked points. For the case $S=T^{2}$ and $n=2$ that is of primary interest to us, the short exact sequence 83 splits [5, 8, 18]:

$$
\mathrm{MCG}_{2}\left(T^{2}\right)=\mathrm{PMCG}_{2}\left(T^{2}\right) \oplus \Sigma_{2}
$$




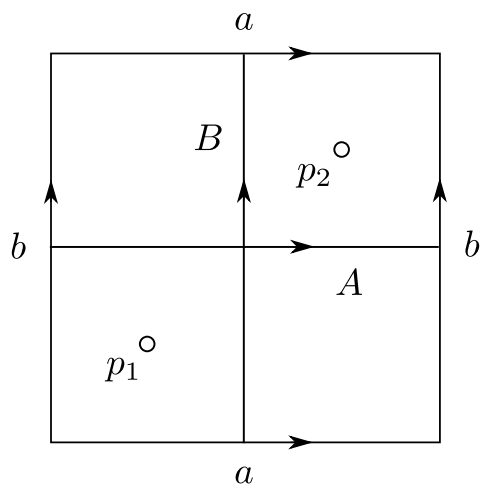

Figure 8. Cycles $a, A, b$, and $B$ corresponding to generators $T_{a}, T_{A}, T_{b}$, and $T_{B}$ of $\mathrm{PMCG}_{2}\left(T^{2}\right)$.

where

$$
\Sigma_{2}=\left\langle\omega \mid \omega^{2}=1\right\rangle=\mathbb{Z}_{2} .
$$

and $\omega$ is represented by a $\pi$-rotation of the square shown in Figure 8 . The pure mapping class group $\mathrm{PMCG}_{2}\left(T^{2}\right)$ is generated by Dehn twists $T_{a}, T_{A}, T_{b}$, and $T_{B}$ around simple closed curves $a, A$, $b$, and $B$ shown in Figure 8, with relations

$$
\begin{aligned}
& T_{a} T_{b}^{-1} T_{a}=T_{b}^{-1} T_{a} T_{b}^{-1}, \\
& T_{A} T_{b}^{-1} T_{A}=T_{b}^{-1} T_{A} T_{b}^{-1}, \\
& T_{a} T_{A}=T_{A} T_{a}, \\
& \left(T_{b}^{-1} T_{a} T_{A}\right)^{4}=1, \\
& T_{B}=T_{a} T_{A}^{-1} T_{b} T_{A} T_{a}^{-1} .
\end{aligned}
$$

\subsection{The Birman sequence for the twice-punctured torus.}

Definition 3.2. Given a surface $S$, we define the configuration space for ordered points $\operatorname{Conf}_{n}^{\prime}(S)$ to be the space

$$
\left\{\left(p_{1}, \cdots, p_{n}\right) \in S^{n} \mid p_{i} \neq p_{j} \text { if } i \neq j\right\} .
$$

We define the configuration space for unordered points $\operatorname{Conf}_{n}(S)$ to be the space $\operatorname{Conf}_{n}^{\prime}(S) / \Sigma_{n}$, where the fundamental group on $n$ letters $\Sigma_{n}$ acts on $\operatorname{Conf}_{n}^{\prime}(S)$ by permutation.

Definition 3.3. Given a surface $S$ and $n$ distinct marked points $p_{1}, \cdots, p_{n} \in S$, we define the braid group $B_{n}(S)$ to be the fundamental group of $\operatorname{Conf}_{n}(S)$ with base point $\left[\left(p_{1}, \cdots, p_{n}\right)\right]$, and we define the pure braid group $P B_{n}(S)$ to be the fundamental group of $\operatorname{Conf}_{n}^{\prime}(S)$ with base point $\left(p_{1}, \cdots, p_{n}\right)$.

There is a short exact sequence

$$
1 \longrightarrow P B_{n}(S) \longrightarrow B_{n}(S) \longrightarrow \Sigma_{n} \longrightarrow 1,
$$

where $\Sigma_{n}$ is the symmetric group of permutations of $\left\{p_{1}, \cdots, p_{n}\right\}$ and the homomorphism $B_{n}(S) \rightarrow \Sigma_{n}$ takes a braid to its corresponding permutation of marked points.

The case $S=T^{2}$ and $n=2$ is of primary interest to us. We can describe a presentation of $B_{2}\left(T^{2}\right)$ as follows 2. Define braids $\alpha_{i}$ and $\beta_{i}$ for $i=1,2$ that drag marked the point $p_{i}$ rightward and upward around a cycle, as shown in Figure 9. Define a braid $\sigma$ that interchanges the marked points via a counterclockwise 


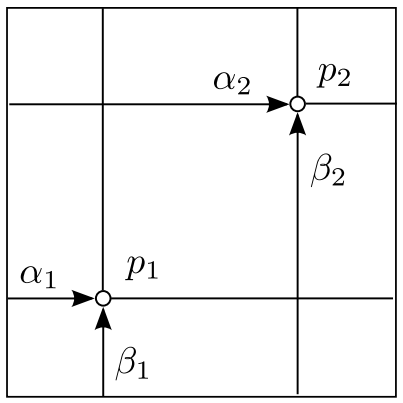

Figure 9. Generators $\alpha_{1}, \alpha_{2}, \beta_{1}$, and $\beta_{2}$ of $B_{2}\left(T^{2}\right)$, which drag the points $p_{1}$ and $p_{2}$ around cycles.

$\pi$-rotation. Then $B_{2}\left(T^{2}\right)$ is generated by $\alpha_{1}, \beta_{1}, \alpha_{2}, \beta_{2}$, and $\sigma$, with relations

$$
\begin{aligned}
& \alpha_{2}=\sigma^{-1} \alpha_{1} \sigma^{-1}, \\
& \beta_{2}=\sigma \beta_{1} \sigma, \\
& \alpha_{1} \alpha_{2}=\alpha_{2} \alpha_{1}, \\
& \beta_{1} \beta_{2}=\beta_{2} \beta_{1}, \\
& \beta_{1}^{-1} \alpha_{1}^{-1} \beta_{1} \alpha_{1}=\sigma^{2}, \\
& \beta_{1}^{-1} \alpha_{2} \beta_{1} \alpha_{2}^{-1}=\sigma^{2} .
\end{aligned}
$$

The braid group $B_{2}\left(T^{2}\right)$ and mapping class group $\mathrm{MCG}_{2}\left(T^{2}\right)$ are related by the Birman exact sequence 3]:

$$
1 \longrightarrow \pi_{1}\left(\text { Homeo }_{0}\left(T^{2}\right)\right) \stackrel{f}{\longrightarrow} B_{2}\left(T^{2}\right) \stackrel{\delta}{\longrightarrow} \mathrm{MCG}_{2}\left(T^{2}\right) \stackrel{g}{\longrightarrow} \mathrm{MCG}\left(T^{2}\right) \longrightarrow 1 .
$$

Here $\mathrm{Homeo}_{0}\left(T^{2}\right)$ is the space of orientation-preserving homeomorphisms of $T^{2}$ that are isotopic to the identity. Since $T^{2}$ acts on itself by translations, and translations are clearly isotopic to the identity, we have that $T^{2} \subset \operatorname{Homeo}_{0}\left(T^{2}\right)$. In fact, one can show that $\operatorname{Homeo}_{0}\left(T^{2}\right)$ deformation retracts onto $T^{2}$ (see [10]), and thus

$$
\pi_{1}\left(\operatorname{Homeo}_{0}\left(T^{2}\right)\right)=\pi_{1}\left(T^{2}\right)=\mathbb{Z}^{2} .
$$

The two free abelian generators of $\pi_{1}\left(\operatorname{Homeo}_{0}\left(T^{2}\right)\right)$ can be identified with the elements $\alpha_{1} \alpha_{2}$ and $\beta_{1} \beta_{2}$ of $B_{2}\left(T^{2}\right)$ under the injection $\pi_{1}\left(\right.$ Homeo $\left._{0}\left(T^{2}\right)\right) \rightarrow B_{2}\left(T^{2}\right)$ in the Birman sequence; these elements can be easily seen to commute using the above presentation for $B_{2}\left(T^{2}\right)$.

The group $\operatorname{MCG}\left(T^{2}\right)$ that appears in the Birman sequence is the mapping class group $\mathrm{MCG}_{0}\left(T^{2}\right)$ of $T^{2}$ with no marked points. Two useful presentations for $\operatorname{MCG}\left(T^{2}\right)$ are (see [7] Section 3.6.4 and [19] page 81):

$$
\operatorname{MCG}\left(T^{2}\right)=\left\langle T_{a}, T_{b} \mid T_{a} T_{b}^{-1} T_{a}=T_{b}^{-1} T_{a} T_{b}^{-1},\left(T_{a} T_{b}^{-1} T_{a}\right)^{4}=1\right\rangle=\left\langle s, t \mid s^{4}=1,(s t)^{3}=s^{2}\right\rangle .
$$

Here $T_{a}$ and $T_{b}$ are Dehn twists around the simple closed curves $a$ and $b$ shown in Figure 8. The two presentations are related by

$$
s=T_{a} T_{b}^{-1} T_{a}=T_{b}^{-1} T_{a} T_{b}^{-1}, \quad t=T_{b} .
$$

It is well-known that $\mathrm{MCG}\left(T^{2}\right)$ is isomorphic to $S L(2, \mathbb{Z})$, where the isomorphism is given by

$$
T_{a} \mapsto\left(\begin{array}{cc}
1 & 1 \\
0 & 1
\end{array}\right), \quad \quad T_{b}=t \mapsto\left(\begin{array}{cc}
1 & 0 \\
1 & 1
\end{array}\right), \quad s \mapsto\left(\begin{array}{cc}
0 & 1 \\
-1 & 0
\end{array}\right) .
$$

We can describe the homomorphisms that appear in the Birman sequence $(99)$ in terms of the group presentations described above. The push homomorphism $\delta: B_{2}\left(T^{2}\right) \rightarrow \mathrm{MCG}_{2}\left(T^{2}\right)$ is given by

$$
\delta\left(\alpha_{1}\right)=\delta\left(\alpha_{2}\right)^{-1}=T_{a} T_{A}^{-1}, \quad \delta\left(\beta_{1}\right)=\delta\left(\beta_{2}\right)^{-1}=T_{b} T_{B}^{-1}, \quad \delta(\sigma)=\left(T_{a} T_{b}^{-1} T_{a}\right)^{2} \omega .
$$

The forgetful homomorphism $g: \mathrm{MCG}_{2}\left(T^{2}\right) \rightarrow \operatorname{MCG}\left(T^{2}\right)$ is given by

$$
g\left(T_{a}\right)=g\left(T_{A}\right)=T_{a}, \quad g\left(T_{b}\right)=g\left(T_{B}\right)=T_{b}, \quad g(\omega)=\left(T_{a} T_{b}^{-1} T_{a}\right)^{2}=s^{2} .
$$


Remark 3.1. It is easy to verify that the composition of two successive maps in the Birman sequence (99) is trivial, and that $\mathrm{MCG}_{2}\left(T^{2}\right) / \operatorname{im} \delta=\operatorname{MCG}\left(T^{2}\right)$, so im $\delta=\operatorname{ker} g$.

\subsection{The action of $\mathrm{MCG}_{2}\left(T^{2}\right)$ on $R\left(T^{2}, 2\right)$.}

Theorem 3.1. Given a surface $S$ and $n$ distinct marked points $p_{1}, \cdots, p_{n} \in S$, there is a homomorphism $\mathrm{MCG}_{n}(S) \rightarrow \operatorname{Out}\left(\pi_{1}\left(S-\left\{p_{1}, \cdots, p_{n}\right\}\right)\right)$.

Proof. Define $X=S-\left\{p_{1}, \cdots, p_{n}\right\}$. Choose a base point $x_{0} \in X$ and consider the fundamental group $\pi_{1}\left(X, x_{0}\right)$. Given an element $[\psi] \in \mathrm{MCG}_{n}(X)$ represented by a homeomorphism $\psi: X \rightarrow X$, there is an induced isomorphism $\psi_{*}: \pi_{1}\left(X, x_{0}\right) \rightarrow \pi_{1}\left(X, \psi\left(x_{0}\right)\right),[\alpha] \mapsto[\psi \circ \alpha]$. Choose a path $\gamma: I \rightarrow X$ from $x_{0}$ to $\psi\left(x_{0}\right)$; this induces an isomorphism $\gamma_{*}: \pi_{1}\left(X, \psi\left(x_{0}\right)\right) \rightarrow \pi_{1}\left(X, x_{0}\right),[\alpha] \mapsto[\gamma \alpha \bar{\gamma}]$. We now define a map $\mathrm{MCG}_{n}(S) \rightarrow \operatorname{Out}\left(\pi_{1}\left(X, x_{0}\right)\right)$ by $[\psi] \mapsto\left[\gamma_{*} \psi_{*}\right]$. One can show that this map is well-defined and is a homomorphism (see [7] Chapter 8.1).

Remark 3.2. A version of the Dehn-Nielsen-Baer theorem states that the homomorphism $\mathrm{MCG}_{n}(S) \rightarrow$ $\operatorname{Out}\left(\pi_{1}\left(S-\left\{p_{1}, \cdots, p_{n}\right\}\right)\right)$ is injective (see [7] Theorem 8.8). This result can be used to check the relations for the presentation of $\mathrm{MCG}_{2}\left(T^{2}\right)$ given above, as well as the expressions for the maps $\delta$ and $g$ in the Birman sequence 99.

We can use Theorem 3.1 to define a right action of $\mathrm{MCG}_{2}\left(T^{2}\right)$ on the character variety $R\left(T^{2}, 2\right)$ by

$$
[\rho] \cdot f=[\rho \circ \phi],
$$

where $[\rho] \in R\left(T^{2}, 2\right), f \in \mathrm{MCG}_{2}\left(T^{2}\right)$, and $\phi \in \operatorname{Aut}\left(\pi_{1}\left(S-\left\{p_{1}, p_{2}\right\}\right)\right)$ is chosen such that $[\phi]$ is the image of $f$ under the homomorphism $\mathrm{MCG}_{2}(\Sigma) \rightarrow \operatorname{Out}\left(\pi_{1}\left(S-\left\{p_{1}, p_{2}\right\}\right)\right)$. The action of $\mathrm{MCG}_{2}\left(T^{2}\right)$ on $R\left(T^{2}, 2\right)$ is given by

$$
\begin{aligned}
& {[(A, B, a, b)] \cdot T_{a}=[(A, B A, a, b)],} \\
& {[(A, B, a, b)] \cdot T_{b}=[(A B, B, a, b)],} \\
& {[(A, B, a, b)] \cdot T_{A}=\left[\left(A, a A B, a, A a b a^{-1} A^{-1}\right)\right],} \\
& {[(A, B, a, b)] \cdot T_{B}=\left[\left(a^{-1} B A, B, a, a^{-1} B b B^{-1} a\right)\right],} \\
& {[(A, B, a, b)] \cdot \omega=\left[\left(A^{-1}, B^{-1}, B^{-1} A^{-1} b A B, A^{-1} B^{-1} a B A\right)\right] .}
\end{aligned}
$$

The homomorphism $\delta: B_{2}\left(T^{2}\right) \rightarrow \mathrm{MCG}_{2}\left(T^{2}\right)$ in the Birman sequence 99 induces a right action of $B_{2}\left(T^{2}\right)$ on $R\left(T^{2}, 2\right)$ that is given by

$$
\begin{aligned}
& {[(A, B, a, b)] \cdot \alpha_{1}=\left[\left(A, a^{-1} B, A a A^{-1}, A a^{-1} A^{-1} b A a A^{-1}\right)\right],} \\
& {[(A, B, a, b)] \cdot \beta_{1}=\left[\left(a A, B, B a B^{-1}, a b a^{-1}\right)\right],} \\
& {[(A, B, a, b)] \cdot \alpha_{2}=\left[\left(A, a b^{-1} a^{-1} B, a, A a b a^{-1} A^{-1}\right)\right],} \\
& {[(A, B, a, b)] \cdot \beta_{2}=\left[\left(b A, B, a, a^{-1} B b B^{-1} a\right)\right],} \\
& {[(A, B, a, b)] \cdot \sigma=\left[\left(A, B, b, b^{-1} a b\right)\right] .}
\end{aligned}
$$

\section{Nondegeneracy}

In this section, we adapt an argument from [1] to obtain a simple criterion for determining when a point $[\rho] \in R_{\pi}^{\natural}(Y, K)$ is nondegenerate; namely, it is nondegenerate if and only if the Lagrangians $L_{s}$ and $L_{2}$ in $R\left(T^{2}, 2\right)$ corresponding to the Heegaard splitting of $Y$ intersect transversely at the image of $[\rho]$ under the pullback map $R_{\pi}^{\natural}(Y, K) \rightarrow R\left(T^{2}, 2\right)$. The argument relies on several results involving group cohomology and the regularity of character varieties, which we discuss first. To make sense of the notion of transversality, we also discuss some results involving the smooth structure of character varieties.

4.1. Constrained group cohomology. Consider a finitely presented group $\Gamma=\langle S \mid R\rangle$ with generators $S=\left\{s_{1}, \cdots, s_{n}\right\}$ and relations $R=\left\{r_{1}, \cdots, r_{m}\right\}$. In defining character varieties, we often want to consider a space $X(\Gamma) \subseteq \operatorname{Hom}(\Gamma, S U(2))$ consisting of homomorphisms that satisfy certain constraints; for example, we may require the homomorphisms to map certain generators to traceless matrices. Provided the constraints are algebraic, the space $X(\Gamma)$ has the structure of a real algebraic variety, and we can define a corresponding scheme $\mathcal{X}(\Gamma)$ whose set of closed points is $X(\Gamma)$. The group $S U(2)$ acts on the variety $X(\Gamma)$ by conjugation, 
and we define the character variety $R(\Gamma)$ and character scheme $\mathcal{R}(\Gamma)$ to be the GIT quotients $X(\Gamma) / / S U(2)$ and $\mathcal{X}(\Gamma) / / S U(2)$. Generalizing a result due to Weil for the unconstrained case [21], we have that the Zariski tangent space $T_{[\rho]} \mathcal{R}(\Gamma)$ of the character scheme $\mathcal{R}(\Gamma)$ at a closed point $[\rho]$ can be identified with the constrained group cohomology $H_{c}^{1}(\Gamma ; \operatorname{Ad} \rho)$, which we define here.

Roughly speaking, the constrained group cohomology $H_{c}^{1}(\Gamma$; Ad $\rho)$ describes deformations of homomorphisms $\rho: \Gamma \rightarrow S U(2)$ that satisfy the relevant constraints, modulo deformations that can be obtained by the conjugation action of $S U(2)$. The precise definition of $H_{c}^{1}(\Gamma ; \mathrm{Ad} \rho)$ that we will use is as follows. Define a function $F_{r}: \operatorname{Hom}(\langle S\rangle, S U(2)) \rightarrow S U(2)^{m}$ by

$$
F_{r}(\rho)=\left(\rho\left(r_{1}\right), \cdots, \rho\left(r_{m}\right)\right)
$$

Thus $F_{r}(\rho)=(1, \cdots, 1)$ if and only if $\rho:\langle S\rangle \rightarrow S U(2)$ preserves all the relations in $R$ and thus descends to a homomorphism $\rho: \Gamma \rightarrow S U(2)$. Given a homomorphism $\rho: \Gamma \rightarrow S U(2)$ and a function $\eta: S \rightarrow \mathfrak{g}$, define a homomorphism $\rho_{t}:\langle S\rangle \rightarrow S U(2)$ such that

$$
\rho_{t}\left(s_{k}\right)=e^{t \eta\left(s_{k}\right)} \rho\left(s_{k}\right) .
$$

Note that we can view $\eta$ as a vector in $\mathfrak{g}^{\oplus n}$. We define a linear map $c_{r}: \mathfrak{g}^{\oplus n} \rightarrow \mathfrak{g}^{\oplus m}$ by

$$
c_{r}(\eta)=\left.\frac{d}{d t} F_{r}\left(\rho_{t}\right)\right|_{t=0} .
$$

Thus $c_{r}(\eta)=0$ if an only if $\eta$ describes a deformation of $\rho$ that is a homomorphism $\Gamma \rightarrow S U(2)$.

Homomorphisms $\Gamma \rightarrow S U(2)$ that represent points in a character variety may be required to satisfy certain constraints; for example, that they take particular generators to traceless matrices. Define a function $F_{c}: \operatorname{Hom}(\langle S\rangle, S U(2)) \rightarrow \mathbb{R}^{q}$ such that $F_{c}(\rho)=0$ if and only if $\rho$ satisfies these constraints; for example, if we require that $\rho$ take the generator $s_{1}$ to a traceless matrix, we would define $F_{c}: \operatorname{Hom}(\langle S\rangle, S U(2)) \rightarrow \mathbb{R}$ by

$$
F_{c}(\rho)=\operatorname{tr}\left(\rho\left(s_{1}\right)\right) .
$$

We define a linear map $c_{c}: \mathfrak{g}^{\oplus n} \rightarrow \mathbb{R}^{q}$ by

$$
c_{c}(\eta)=\left.\frac{d}{d t} F_{c}\left(\rho_{t}\right)\right|_{t=0}
$$

Thus $c_{c}(\eta)=0$ if and only if $\eta$ describes a deformation of $\rho$ that satisfies the constraints.

We now combine the linear maps for the relations and constraints to obtain a linear map $c: \mathfrak{g}^{\oplus n} \rightarrow$ $\mathfrak{g}^{\oplus m} \oplus \mathbb{R}^{q}, c(\eta)=\left(c_{r}(\eta), c_{c}(\eta)\right)$. Given a homomorphism $\rho: \Gamma \rightarrow S U(2)$ that satisfies the constraints, we define the space of 1-cocycles to be

$$
Z_{c}^{1}(\Gamma ; \operatorname{Ad} \rho)=\operatorname{ker} c
$$

so a vector $\eta \in \mathfrak{g}^{n}$ is a 1-cocycle if and only if it describes a deformation of $\rho$ that is a homomorphism that preserves the constraints. We define the space of 1-coboundaries to be deformations of $\rho$ that are obtained via the conjugation action of $S U(2)$ :

$$
B_{c}^{1}(\Gamma ; \operatorname{Ad} \rho)=\left\{\eta: S \rightarrow \mathfrak{g} \mid \text { there exists } u \in \mathfrak{g} \text { such that } \eta\left(s_{k}\right)=u-\operatorname{Ad}_{\rho\left(s_{k}\right)} u \text { for } k=1, \cdots, n\right\} .
$$

We define the constrained group cohomology $H_{c}^{1}(\Gamma ; \operatorname{Ad} \rho)$ to be

$$
H_{c}^{1}(\Gamma ; \operatorname{Ad} \rho)=Z_{c}^{1}(\Gamma ; \operatorname{Ad} \rho) / B_{c}^{1}(\Gamma ; \operatorname{Ad} \rho) .
$$

\subsection{Regularity.}

Definition 4.1. We say that a point $[\rho]$ of a character variety $R(\Gamma)$ is regular $\operatorname{if} \operatorname{dim}_{[\rho]} R(\Gamma)=\operatorname{dim} H_{c}^{1}(\Gamma ; \operatorname{Ad} \rho)$.

We can define a space of regular points $R^{\prime}(\Gamma) \subseteq R(\Gamma)$ for which $\operatorname{dim}_{[\rho]} R(\Gamma)=\operatorname{dim} H_{c}^{1}(\Gamma ; \operatorname{Ad} \rho)$. The space $R^{\prime}(\Gamma)$ has the structure of a smooth manifold, and the tangent space of this manifold at a point $[\rho] \in R^{\prime}(\Gamma)$ is given by $T_{[\rho]} R^{\prime}(\Gamma)=H_{c}^{1}(\Gamma ; \operatorname{Ad} \rho)$.

Theorem 4.1. The character variety $R\left(S^{1} \times D^{2}, A_{1}\right)$ is regular at all points represented by nonabelian homomorphisms. 
Proof. Using results from the proof of Theorem 2.4, we find that we can take the set of generators of the fundamental group $\Gamma$ to be $S=\{A, a\}$, with no relations, and we can take the constraint function $F_{c}: \operatorname{Hom}(\langle S\rangle, S U(2)) \rightarrow \mathbb{R}$ to be

$$
F_{c}(\rho)=\operatorname{tr}(\rho(a)) .
$$

Using equation 125, together with the expressions for the homomorphisms $\rho: \Gamma \rightarrow S U(2)$ given in Theorem 2.4. we obtain a linear map $c: \mathbb{R}^{6} \rightarrow \mathbb{R}$. A straightforward calculation shows that $\operatorname{dim} H_{c}^{1}(\Gamma ; \operatorname{Ad} \rho)=$ $\operatorname{dim} R\left(S^{1} \times D^{2}, A_{1}\right)=2$ for all $[\rho] \in R\left(S^{1} \times D^{2}, A_{1}\right)$ such that $\rho$ is nonabelian.

We would next like to determine the regular points of the perturbed character variety $R_{\pi}^{\natural}\left(S^{1} \times D^{2}, A_{1}\right)$, but there are two difficulties that must be overcome. The first difficulty involves the function $f(\phi)$ that defines the perturbation. Recall that points $[\rho] \in R_{\pi}^{\natural}\left(S^{1} \times D^{2}, A_{1}\right)$ are constrained by the requirement that if $\rho\left(\lambda_{P}\right)$ has the form $\rho\left(\lambda_{P}\right)=e^{i \phi \hat{r} \cdot \vec{\sigma}}$, then $\rho\left(\mu_{P}\right)$ must have the form $\rho\left(\lambda_{P}\right)=e^{i \nu \hat{r} \cdot \vec{\sigma}}$, where $\nu=\epsilon f(\phi)$. In order to give $R_{\pi}^{\natural}\left(S^{1} \times D^{2}, A_{1}\right)$ the structure of a real algebraic variety, and to define the corresponding character scheme, this constraint must be algebraic. We will therefore choose $f(\phi)$ to be

$$
f(\phi)=\frac{1}{\epsilon} \sin ^{-1}(\epsilon \sin \phi) .
$$

Then the constraint on $\rho$ becomes

$$
\epsilon \operatorname{tr}\left(\rho\left(\lambda_{P}\right) \sigma_{i}\right)=\operatorname{tr}\left(\rho\left(\mu_{P}\right) \sigma_{i}\right)
$$

for $i=x, y, z$.

Remark 4.1. In fact, the constraint given in equation (127) yields a variety with two connected components, one with $\rho\left(\mu_{P}\right)$ near 1 and one with $\rho\left(\mu_{P}\right)$ near -1 , and only the first component corresponds to $R_{\pi}^{\natural}\left(S^{1} \times D^{2}, A_{1}\right)$. To calculate the constrained group cohomology, however, we consider only infinitesimal deformations of homomorphisms, hence the extraneous second component is irrelevant.

A second difficulty in determining the regular points of $R_{\pi}^{\natural}\left(S^{1} \times D^{2}, A_{1}\right)$ is that a direct calculation of the constrained group cohomology for $R_{\pi}^{\natural}\left(S^{1} \times D^{2}, A_{1}\right)$ does not appear to be practical, because the perturbed representations, as described in Theorem 2.8 , are rather complicated. Instead, we will apply the following theorem, which simplifies the necessary calculations by allowing us to extrapolate from unperturbed representations:

Theorem 4.2. Consider a character variety $R_{\epsilon}(\Gamma)$ in which the homomorphisms are required to satisfy an algebraic constraint that depends on a control parameter $\epsilon \in \mathbb{R}$. Given a homomorphism $\rho_{\epsilon}: \Gamma \rightarrow S U(2)$ representing a point $\left[\rho_{\epsilon}\right] \in R_{\epsilon}(\Gamma)$, let $c_{\epsilon}: \mathfrak{g}^{\oplus n} \rightarrow \mathfrak{g}^{\oplus m} \oplus \mathbb{R}^{q}$ denote the corresponding linear map used to define the constrained group cohomology. Define $c_{0}, c_{1}: \mathfrak{g}^{\oplus n} \rightarrow \mathfrak{g}^{\oplus m} \oplus \mathbb{R}^{q}$ such that $c_{\epsilon}=c_{0}+\epsilon c_{1}+\cdots$. The following string of inequalities holds for $\epsilon>0$ sufficiently small:

$$
\operatorname{dim} Z_{c}^{1}\left(\Gamma ; \operatorname{Ad} \rho_{\epsilon}\right) \leq \operatorname{dim}\left(\operatorname{ker} c_{0} \cap \operatorname{ker} c_{1}\right)+\operatorname{dim}\left(c_{1}\left(\operatorname{ker} c_{0}\right) \cap \operatorname{im} c_{0}\right) \leq \operatorname{dim} Z_{c}^{1}\left(\Gamma ; \operatorname{Ad} \rho_{0}\right) .
$$

Proof. Since the dimension of the Zariski tangent space is upper semi-continuous, for $\epsilon>0$ sufficiently small we have that

$$
\operatorname{dim}\left(\operatorname{ker} c_{\epsilon}\right)=\operatorname{dim} Z_{c}^{1}\left(\Gamma ; \operatorname{Ad} \rho_{\epsilon}\right) \leq \operatorname{dim} Z_{c}^{1}\left(\Gamma ; \operatorname{Ad} \rho_{0}\right)=\operatorname{dim}\left(\operatorname{ker} c_{0}\right) .
$$

Thus any vector $w_{\epsilon} \in \operatorname{ker} c_{\epsilon}$ must have the form $w_{\epsilon}=w_{0}+\epsilon w_{1}+\cdots$, where

$$
c_{\epsilon}\left(w_{\epsilon}\right)=c_{0}\left(w_{0}\right)+\epsilon\left(c_{0}\left(w_{1}\right)+c_{1}\left(w_{0}\right)\right)+\cdots=0 .
$$

The space of vectors $w_{0} \in \mathfrak{g}^{\oplus n}$ that satisfy equation 130 up to first order in $\epsilon$ is

$$
V=\left\{w_{0} \in \operatorname{ker} c_{0} \mid c_{1}\left(w_{0}\right) \in \operatorname{im} c_{0}\right\} .
$$

Since $\operatorname{ker} c_{\epsilon}=Z_{c}^{1}\left(\Gamma ; \operatorname{Ad} \rho_{\epsilon}\right)$ is the space of vectors that satisfies equation 130 to all orders in $\epsilon$, it follows that $Z_{c}^{1}\left(\Gamma ; \operatorname{Ad} \rho_{\epsilon}\right) \subseteq V \subseteq \operatorname{ker} c_{0}=Z_{c}^{1}\left(\Gamma ; \operatorname{Ad} \rho_{0}\right)$, and we have the string of inequalities

$$
\operatorname{dim} Z_{c}^{1}\left(\Gamma ; \operatorname{Ad} \rho_{\epsilon}\right) \leq \operatorname{dim} V \leq \operatorname{dim} Z_{c}^{1}\left(\Gamma ; \operatorname{Ad} \rho_{0}\right) .
$$

Equation 128 now follows from the fact that

$$
\operatorname{dim} V=\operatorname{dim}\left(\operatorname{ker} c_{0} \cap \operatorname{ker} c_{1}\right)+\operatorname{dim}\left(c_{1}\left(\operatorname{ker} c_{0}\right) \cap \operatorname{im} c_{0}\right) .
$$


Example 4.1. Take $\Gamma=\mathbb{Z}$, and consider the character varieties $R_{\epsilon}^{i}(\Gamma)$ for $i=1,2,3$ with constraint functions $F_{c}^{i}: \operatorname{Hom}(\Gamma, S U(2)) \rightarrow \mathbb{R}$ given by

$$
F_{c}^{1}(\rho)=\epsilon \operatorname{tr} \rho(1), \quad F_{c}^{2}(\rho)=\epsilon(\operatorname{tr} \rho(1))^{2}, \quad F_{c}^{3}(\rho)=\epsilon(\operatorname{tr} \rho(1))^{2}+\epsilon^{2} \operatorname{tr} \rho(1) .
$$

The character varieties are given by

$$
R_{\epsilon}^{1}(\Gamma)=R_{\epsilon}^{2}(\Gamma)=R_{\epsilon}^{3}(\Gamma)= \begin{cases}S^{2} & \text { if } \epsilon \neq 0 \\ S^{3} & \text { if } \epsilon=0\end{cases}
$$

Consider the homomorphism $\rho_{\epsilon}: \mathbb{Z} \rightarrow S U(2), \rho_{\epsilon}(1)=i \sigma_{z}$. Then $\operatorname{dim} Z_{c}^{1}\left(\Gamma ; \operatorname{Ad} \rho_{\epsilon}\right)$ and $\operatorname{dim}\left(\operatorname{ker} c_{0} \cap \operatorname{ker} c_{1}\right)+$ $\operatorname{dim}\left(c_{1}\left(\operatorname{ker} c_{0}\right) \cap \operatorname{im} c_{0}\right)$ are given by

$$
\begin{array}{cccc} 
& F_{c}^{1} & F_{c}^{2} & F_{c}^{3} \\
\operatorname{dim} Z_{c}^{1}\left(\Gamma ; \operatorname{Ad} \rho_{\epsilon}\right) & 2 & 3 & 2 \\
\operatorname{dim}\left(\operatorname{ker} c_{0} \cap \operatorname{ker} c_{1}\right)+\operatorname{dim}\left(c_{1}\left(\operatorname{ker} c_{0}\right) \cap \operatorname{im} c_{0}\right) & 2 & 3 & 3
\end{array}
$$

From the expressions for $\operatorname{dim} Z_{c}^{1}\left(\Gamma ; \operatorname{Ad} \rho_{\epsilon}\right)$, we find that for $\epsilon \neq 0$ the character schemes $\mathcal{R}_{\epsilon}^{1}(\Gamma)$ and $\mathcal{R}_{\epsilon}^{3}(\Gamma)$ are reduced, and the character scheme $\mathcal{R}_{\epsilon}^{2}(\Gamma)$ is not reduced. We can use Theorem 4.2 to show that $\mathcal{R}_{\epsilon}^{1}(\Gamma)$ is reduced, but not that $\mathcal{R}_{\epsilon}^{3}(\Gamma)$ is reduced.

Theorem 4.3. The character variety $R_{\pi}^{\natural}\left(S^{1} \times D^{2}, A_{1}\right)$ is regular everywhere.

Proof. Using results from the proof of Theorem 2.8, we find that we can take the set of generators for the fundamental group $\Gamma$ to be $S=\{a, A, B, h\}$, the relations function $F_{r}: \operatorname{Hom}(\langle S\rangle, S U(2)) \rightarrow S U(2)$ to be

$$
F_{r}(\rho)=-\rho([h, a B]),
$$

and the constraint function $F_{c}: \operatorname{Hom}(\langle S\rangle, S U(2)) \rightarrow \mathbb{R}^{6}$ to be

$$
F_{c}(\rho)=\left(\operatorname{tr}(\rho(a)), \operatorname{tr}\left(\rho\left(h a^{-1} h^{-1}\right)\right), \operatorname{tr}(\rho(h)), f_{x}(\rho), f_{y}(\rho), f_{z}(\rho)\right)
$$

where

$$
f_{i}(\rho)=\epsilon \operatorname{tr}\left(\rho\left(h^{-1} A\right) \sigma_{i}\right)-\operatorname{tr}\left(\rho(B) \sigma_{i}\right) .
$$

Using equations (137) and (138), together with the expressions for the homomorphisms $\rho_{\epsilon}: \Gamma \rightarrow S U(2)$ given in Theorem 2.8, we obtain a linear map $c_{\epsilon}: \mathbb{R}^{12} \rightarrow \mathbb{R}^{9}$. We now apply Theorem 4.2 . A straightforward, but rather lengthy, calculation shows that $\operatorname{dim}\left(\operatorname{ker} c_{0} \cap \operatorname{ker} c_{1}\right)+\operatorname{dim}\left(c_{1}\left(\operatorname{ker} c_{0}\right) \cap \operatorname{im} c_{0}\right)=5$ for all homomorphisms representing points in $R_{\pi}^{\natural}\left(S^{1} \times D^{2}, A_{1}\right)$. Since these homomorphisms are all nonabelian, we conclude that $\operatorname{dim} H_{c}^{1}(\Gamma ; \operatorname{Ad} \rho)=\operatorname{dim} R_{\pi}^{\natural}\left(S^{1} \times D^{2}, A_{1}\right)=2$ for all $[\rho] \in R_{\pi}^{\natural}\left(S^{1} \times D^{2}, A_{1}\right)$, and thus $R_{\pi}^{\natural}\left(S^{1} \times D^{2}, A_{1}\right)$ is regular everywhere.

Theorem 4.4. The character variety $R\left(T^{2}, 2\right)$ is regular on $L_{s}$.

Proof. Using results from Section 2.1 we find that we can take the set of generators for the fundamental group $\Gamma$ to be $S=\{a, A, B\}$, with no relations, and we can take the constraint function $F_{c}: \operatorname{Hom}(\langle S\rangle, S U(2)) \rightarrow \mathbb{R}^{2}$ to be

$$
F_{c}(\rho)=(\operatorname{tr}(\rho(a)), \operatorname{tr}(\rho([A, B] a)) .
$$

Using equation 140 and Theorem 2.8 we obtain a linear map $c_{\epsilon}: \mathbb{R}^{9} \rightarrow \mathbb{R}^{2}$ for homomorphisms representing points in $L_{s}$. A straightforward, but rather lengthy, calculation shows that $\operatorname{dim}\left(\operatorname{ker} c_{0} \cap \operatorname{ker} c_{1}\right)+$ $\operatorname{dim}\left(c_{1}\left(\operatorname{ker} c_{0}\right) \cap \operatorname{im} c_{0}\right)=7$ for all homomorphisms representing points in $L_{s}$. Since these homomorphisms are all nonabelian, we conclude that $\operatorname{dim} H_{c}^{1}(\Gamma ; \operatorname{Ad} \rho)=\operatorname{dim} R\left(T^{2}, 2\right)=4$ for all $[\rho] \in R\left(T^{2}, 2\right)$, and thus $R\left(T^{2}, 2\right)$ is regular on $L_{s}$.

Remark 4.2. We conjecture that $R\left(T^{2}, 2\right)$ is in fact regular at all points represented by nonabelian homomorphisms, but Theorem 4.4 will suffice for our purposes. 


\subsection{Smoothness.}

Remark 4.3. Using simple arguments, which we omit for the sake of brevity, we obtain the following results:

(1) Given a character variety $R(\Gamma)$, we can define functions $R(\Gamma) \rightarrow \mathbb{R}$ of the form $[\rho] \mapsto \operatorname{tr}(\rho(\gamma))$ for $\gamma \in \Gamma$. Such functions are smooth where $R(\Gamma)$ is regular.

(2) The homeomorphism $S^{2} \rightarrow R_{\pi}^{\natural}\left(S^{1} \times D^{2}\right)$ is smooth.

(3) The homeomorphism $P_{4} \rightarrow S^{2} \times S^{2}-\Delta$ is smooth where $P_{4}$ is regular.

(4) The functions $\alpha, \beta: P_{3} \rightarrow \mathbb{R}$ are smooth where $P_{3}$ is regular.

Theorem 4.5. The map $R_{\pi}^{\natural}\left(S^{1} \times D^{2}\right) \rightarrow R\left(T^{2}, 2\right)$ is an immersion away from $z= \pm 1$ and $x= \pm 1$.

Proof. We can define functions $f, g$, and $h$ on suitable open subsets of $R\left(T^{2}, 2\right)$ by

$$
f([\rho])=\operatorname{Arg}(-\operatorname{tr} A B+(i / 2)(\operatorname{tr} B)(\operatorname{tr} A)), \quad g([\rho])=-\frac{\operatorname{tr}(A a)}{\operatorname{tr}(A b)}, \quad h([\rho])=\operatorname{tr} A .
$$

A straightforward calculation shows that the composition of $S^{2} \cap\{y \neq 0\} \rightarrow R_{\pi}^{\natural}\left(S^{1} \times D^{2}\right) \rightarrow R\left(T^{2}, 2\right)$ with $R\left(T^{2}, 2\right) \rightarrow \mathbb{R}^{2},[\rho] \mapsto(f([\rho]), g([\rho]))$ is an immersion, and the composition of $S^{2} \cap\{z \neq \pm 1\} \rightarrow$ $R_{\pi}^{\natural}\left(S^{1} \times D^{2}\right) \rightarrow R\left(T^{2}, 2\right)$ with $R\left(T^{2}, 2\right) \rightarrow \mathbb{R}^{2},[\rho] \mapsto(f([\rho]), h([\rho]))$ is an immersion on $\{y=0, x \neq \pm 1\}$.

Corollary 4.1. The homeomorphism $S^{2} \rightarrow R_{\pi}^{\natural}\left(S^{1} \times D^{2}\right)$ is an immersion away from $z= \pm 1$ and $x= \pm 1$.

Proof. This follows from the proof of Theorem 4.5

Remark 4.4. In fact, a slightly more complicated argument shows that the maps described in Theorem 4.5 and Corollary 4.1 are also immersions at $x= \pm 1$.

Theorem 4.6. The following composition of maps is an immersion for $\epsilon>0$ sufficiently small:

$$
S^{2} \cap\{y \neq 0\} \rightarrow R_{\pi}^{\natural}\left(S^{1} \times D^{2}\right) \cap\{y \neq 0\} \rightarrow P_{4} \rightarrow S^{2} \times S^{2}-\Delta .
$$

Proof. This is a straightforward calculation using the expressions for $\hat{a}\left(L_{s}(\phi, \theta)\right)$ and $\hat{b}\left(L_{s}(\phi, \theta)\right)$ given in equations (70)- 77.

4.4. Transversality. We are now ready to prove our key result that relates nondegeneracy to transversality. The following is a restatement of Theorem 1.4 from the introduction:

Theorem 4.7. Assume the map $R\left(U_{2}, A_{2}\right) \rightarrow R\left(T^{2}, 2\right)$ is an injective immersion; recall that we denote the image of this map by $L_{2}$. Consider a point $[\rho] \in L_{s} \cap L_{2} \subset R\left(T^{2}, 2\right)$ that is the image of a regular point of $R\left(U_{2}, A_{2}\right)$ and is not the double-point of $L_{s}$. By Corollary 1.1, the point [ $\rho$ ] is the image of a unique point in $R_{\pi}^{\natural}(Y, K)$ under the pullback map $R_{\pi}^{\natural}(Y, K) \rightarrow R\left(T^{2}, 2\right)$, which for simplicity we also denote by $[\rho]$. The point $[\rho] \in R_{\pi}^{\natural}(Y, K)$ is nondegenerate if and only if the intersection of $L_{s}$ with $L_{2}$ at $[\rho] \in R\left(T^{2}, 2\right)$ is transverse.

Proof. We introduce the notation $K^{\prime}=K \cup W \cup H \cup P, Y^{\prime}=Y-K^{\prime}, U_{i}^{\prime}=U_{i}-K^{\prime}$, and $\Sigma^{\prime}=T^{2}-\left\{p_{1}, p_{2}\right\}$. We have the following Mayer-Vietoris sequence:

$$
\cdots \longrightarrow H_{c}^{0}\left(\Sigma^{\prime} ; \operatorname{Ad} \rho\right) \longrightarrow H_{c}^{1}\left(Y^{\prime} ; \operatorname{Ad} \rho\right) \longrightarrow H_{c}^{1}\left(U_{1}^{\prime} ; \operatorname{Ad} \rho\right) \oplus H_{c}^{1}\left(U_{2}^{\prime} ; \operatorname{Ad} \rho\right) \longrightarrow H_{c}^{1}\left(\Sigma^{\prime} ; \operatorname{Ad} \rho\right) \longrightarrow \cdots
$$

Here $H_{c}^{0}\left(\Sigma^{\prime} ; \operatorname{Ad} \rho\right)$ is

$$
H_{c}^{0}\left(\Sigma^{\prime} ; \operatorname{Ad} \rho\right)=\left\{x \in \mathfrak{g} \mid[\rho(\lambda), x]=0 \text { for all } \lambda \in \pi_{1}\left(\Sigma^{\prime}\right)\right\}
$$

and $H_{c}^{1}\left(Y^{\prime} ; \operatorname{Ad} \rho\right), H_{c}^{1}\left(U_{1}^{\prime} ; \operatorname{Ad} \rho\right), H_{c}^{1}\left(U_{2}^{\prime} ; \operatorname{Ad} \rho\right), H_{c}^{1}\left(\Sigma^{\prime} ; \operatorname{Ad} \rho\right)$ are the constrained group cohomology for the character varieties $R_{\pi}^{\natural}(Y, K), R_{\pi}^{\natural}\left(U_{1}, A_{1}\right), R\left(U_{2}, A_{2}\right)$, and $R\left(T^{2}, 2\right)$, respectively. We are being sloppy and using $\rho$ to denote a homomorphism representing a point in $R_{\pi}^{\natural}(Y, K)$, as well as its pullbacks to homomorphisms representing points in $R_{\pi}^{\natural}\left(U_{1}, A_{1}\right), R\left(U_{2}, A_{2}\right)$, and $R\left(T^{2}, 2\right)$. Since all points in $L_{s}$ are represented by nonabelian homomorphisms, we have that $H_{c}^{0}\left(\Sigma^{\prime} ; \operatorname{Ad} \rho\right)=0$. From Theorems 4.3 and 4.4 , we have the identifications

$$
H_{c}^{1}\left(U_{1}^{\prime} ; \operatorname{Ad} \rho\right)=T_{[\rho]} R_{\pi}^{\natural}\left(S^{1} \times D^{2}, A_{1}\right), \quad H_{c}^{1}\left(\Sigma^{\prime} ; \operatorname{Ad} \rho\right)=T_{[\rho]} R\left(T^{2}, 2\right) .
$$

Since we have assumed that $[\rho] \in R\left(U_{2}, A_{2}\right)$ is regular, we have the identification

$$
H_{c}^{1}\left(U_{2}^{\prime} ; \operatorname{Ad} \rho\right)=T_{[\rho]} R\left(U_{2}, A_{2}\right) .
$$




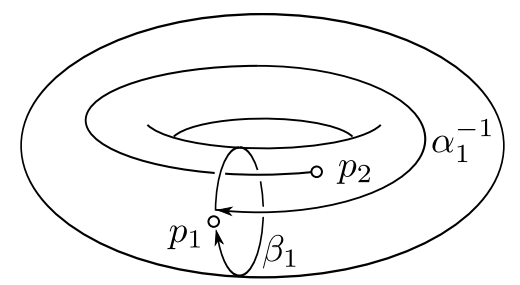

Figure 10. The mapping class group element $f=s \beta_{1} \alpha_{1}^{-1}$ makes the trefoil in $S^{3}$.

By Theorem 4.5 the map $R_{\pi}^{\natural}\left(S^{1} \times D^{2}, A_{1}\right) \rightarrow R\left(T^{2}, 2\right)$ is an immersion (with image $L_{s}$ ), and we have assumed that $R\left(U_{2}, A_{2}\right) \rightarrow R\left(T^{2}, 2\right)$ is an immersion (with image $L_{2}$ ), so we can identify

$$
T_{[\rho]} R_{\pi}^{\natural}\left(S^{1} \times D^{2}, A_{1}\right)=T_{[\rho]} L_{s}, \quad T_{[\rho]} R_{\pi}^{\natural}\left(U_{2}, A_{2}\right)=T_{[\rho]} L_{2} .
$$

We conclude that the constrained group cohomology $H_{c}^{1}\left(Y^{\prime} ; \operatorname{Ad} \rho\right)$ is given by

$$
H_{c}^{1}\left(Y^{\prime} ; \operatorname{Ad} \rho\right)=T_{[\rho]} \mathcal{R}_{\pi}^{\natural}(Y, K)=T_{[\rho]} L_{s} \cap T_{[\rho]} L_{2} .
$$

The constrained group cohomology $H_{c}^{1}\left(Y^{\prime} ; \operatorname{Ad} \rho\right)$ is zero if and only if $[\rho]$ is nondegenerate (see [6] Section 2.5.4). Thus $[\rho]$ is nondegenerate if and only if $L_{s}$ intersects $L_{2}$ transversely at $[\rho]$.

Example 4.2. Consider the algebraic functions $f, g: \mathbb{R} \rightarrow \mathbb{R}, f(x)=x^{2}, g(x)=x^{3}$. The schemes corresponding to the critical loci of $f$ and $g$ are $\operatorname{Spec} F=\{(0)\}$ and $\operatorname{Spec} G=\{(x)\}$, where

$$
F=\mathbb{R}[x] /\left(f^{\prime}(x)\right)=\mathbb{R}, \quad G=\mathbb{R}[x] /\left(g^{\prime}(x)\right)=\mathbb{R}[x] /\left(x^{2}\right) .
$$

The fact that 0 is a nondegenerate critical point of $f$, but a degenerate critical point of $g$, is reflected in the fact that $F$ is reduced, but $G$ is nonreduced, which in turn is reflected in the fact that $T_{(0)} \operatorname{Spec} F=0$, but $T_{(x)} \operatorname{Spec} G=\mathbb{R}$.

Remark 4.5. Since nondegeneracy is a stable property, for sufficiently small $\epsilon>0$ we can use the function $f(\phi)=\sin \phi$ to define the perturbation, rather than the function $f(\phi)$ given in equation (126).

\section{EXAmples}

5.1. Trefoil in $S^{3}$. As shown in Figure 10, we can make the trefoil in $S^{3}$ by taking the element of the mapping class group to be $f=s \beta_{1} \alpha_{1}^{-1} \in \mathrm{MCG}_{2}\left(T^{2}\right)$. We first prove a result that constrains the possible intersection points of $L_{s}$ and $L_{2}=L_{d} \cdot f$ :

Lemma 5.1. If $L_{d}(\chi, \psi) \cdot f=L_{s}(\phi, \theta)$, then $\chi=\pi / 2$ and either $\theta \in\{\pi / 2,3 \pi / 2\}$ or $\phi \in\{0, \pi\}$

Proof. Define functions $h_{1}, h_{2}: R\left(T^{2}, 2\right) \rightarrow \mathbb{R}$ by

$$
h_{1}([\rho])=\operatorname{tr} A, \quad h_{2}([\rho])=\operatorname{tr} B a .
$$

We find that

$$
\begin{array}{ll}
h_{1}\left(L_{s}(\phi, \theta)\right)=-\frac{2 \cos \nu \cos \theta \sin \phi}{\sqrt{\cos ^{2} \nu+\sin ^{2} \nu \sin ^{2} \theta}}, & h_{1}\left(L_{d}(\chi, \psi) \cdot f\right)=0, \\
h_{2}\left(L_{s}(\phi, \theta)\right)=0, & h_{2}\left(L_{d}(\chi, \psi) \cdot f\right)=-2 \cos \chi .
\end{array}
$$

The result follows from equations 150 and 151 .

Theorem 5.1. The rank of the singular instanton homology for the trefoil in $S^{3}$ is at most 3.

Proof. For $\chi=\pi / 2$ we find that $L_{d}(\chi, \psi) \cdot f=[(A, B, a, b)]$, where

$$
A=i \sigma_{x}, \quad B=\sin 3 \psi-i \sigma_{z} \cos 3 \psi, \quad a=i \vec{\sigma} \cdot \hat{a}, \quad b=i \vec{\sigma} \cdot \hat{b},
$$

and

$$
\hat{a}=(-\cos 2 \psi, \sin 2 \psi, 0), \quad \hat{b}=(-\cos 4 \psi,-\sin 4 \psi, 0) .
$$




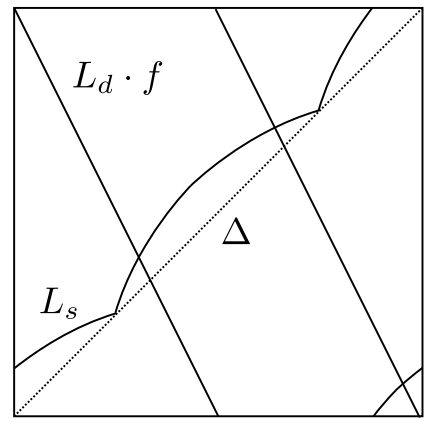

Figure 11. The trefoil in $S^{3}$, with $f=s \beta_{1} \alpha_{1}^{-1}$. The space depicted is $T^{2} \subset S^{2} \times S^{2}$. Shown are the sphere Lagrangian $L_{s}$, the disk Lagrangian $L_{d} \cdot f$, and the diagonal $\Delta$.

We see that if $\cos 3 \psi=0$, corresponding to $\psi \in\{ \pm \pi / 6, \pm \pi / 2\}$, then the point $L_{d}(\chi, \psi) \cdot f$ lies in the piece $P_{3}$, otherwise it lies in the piece $P_{4}$. We first consider the portion of $L_{d} \cdot f$ that lies in the piece $P_{3}$. From equations $(152)$ and $(153)$, we find that

$$
\begin{array}{llll}
(\chi, \psi)=(\pi / 2, \pi / 6): & \alpha\left(L_{d}(\chi, \psi) \cdot f\right)=\pi / 2, & \beta\left(L_{d}(\chi, \psi) \cdot f\right)=0, & \gamma\left(L_{d}(\chi, \psi) \cdot f\right)=-\pi / 6, \\
(\chi, \psi)=(\pi / 2,-\pi / 6): & \alpha\left(L_{d}(\chi, \psi) \cdot f\right)=\pi / 2, & \beta\left(L_{d}(\chi, \psi) \cdot f\right)=\pi, & \gamma\left(L_{d}(\chi, \psi) \cdot f\right)=-\pi / 6, \\
(\chi, \psi)=(\pi / 2, \pi / 2): & \alpha\left(L_{d}(\chi, \psi) \cdot f\right)=\pi / 2, & \beta\left(L_{d}(\chi, \psi) \cdot f\right)=\pi, & \gamma\left(L_{d}(\chi, \psi) \cdot f\right)=\pi / 2, \\
(\chi, \psi)=(\pi / 2,-\pi / 2): & \alpha\left(L_{d}(\chi, \psi) \cdot f\right)=\pi / 2, & \beta\left(L_{d}(\chi, \psi) \cdot f\right)=0, & \gamma\left(L_{d}(\chi, \psi) \cdot f\right)=\pi / 2 .
\end{array}
$$

From Theorem 2.10, it follows that none of these four points lie in $L_{s}$. Next we consider the portion of $L_{d} \cdot f$ that lies in the piece $P_{4}$. From equations 152 and 153 , we find that

$$
\chi=\pi / 2: \quad \hat{a}\left(L_{d}(\chi, \psi) \cdot f\right)=(-\cos 2 \psi, \sin 2 \psi, 0), \quad \hat{b}\left(L_{d}(\chi, \psi) \cdot f\right)=(-\cos 4 \psi,-\sin 4 \psi, 0) .
$$

We substitute $\theta=\pi / 2$ and $\theta=3 \pi / 2$ into equations $70-77$ for $\hat{a}\left(L_{s}(\phi, \theta)\right)$ and $\hat{b}\left(L_{s}(\phi, \theta)\right)$ to obtain

$$
\theta=\pi / 2: \quad \hat{a}\left(L_{s}(\phi, \theta)\right)=(-\sin (\phi+\nu),-\cos (\phi+\nu), 0), \quad \hat{b}\left(L_{s}(\phi, \theta)\right)=(\sin (\phi-\nu), \cos (\phi-\nu), 0),
$$

$$
\theta=3 \pi / 2: \quad \hat{a}\left(L_{s}(\phi, \theta)\right)=(\sin (\phi+\nu), \cos (\phi+\nu), 0), \quad \hat{b}\left(L_{s}(\phi, \theta)\right)=(-\sin (\phi-\nu),-\cos (\phi-\nu), 0) .
$$

From equations (158), it follows that the intersection of $L_{d} \cdot f$ with $L_{s}$ in $P_{4}$ in fact takes place in a torus $T^{2}-\Delta \subset S^{2} \times S^{2}-\Delta$, where $\Delta \subset T^{2}$ is the diagonal. Using equations (158), (159), and (160), we plot the intersection of $L_{d} \cdot f$ and $L_{s}$ in $T^{2}-\Delta$ in Figure 11. We conclude that $L_{d} \cdot f$ and $L_{s}$ intersect in three points.

We will now show that the intersection is transverse at each of these points. At each point we find that

$$
\begin{array}{llll}
\partial_{\phi} h_{1}\left(L_{s}(\phi, \theta)\right)=0, & \partial_{\theta} h_{1}\left(L_{s}(\phi, \theta)\right) \neq 0, & \partial_{\chi} h_{1}\left(L_{d}(\chi, \psi) \cdot f\right)=0, & \partial_{\psi} h_{1}\left(L_{d}(\chi, \psi) \cdot f\right)=0, \\
\partial_{\phi} h_{2}\left(L_{s}(\phi, \theta)\right)=0, & \partial_{\theta} h_{2}\left(L_{s}(\phi, \theta)\right)=0, & \partial_{\chi} h_{2}\left(L_{d}(\chi, \psi) \cdot f\right) \neq 0, & \partial_{\psi} h_{2}\left(L_{d}(\chi, \psi) \cdot f\right)=0 .
\end{array}
$$

Equations (158)- 162 , together with Figure 11, show that the intersection is transverse at each intersection point.

Remark 5.1. The reduced Khovanov homology of the trefoil in $S^{3}$ has rank 3, so Theorem 5.1, in conjunction with the spectral sequence from singular instanton homology to reduced Khovanov homology, amounts to a calculation of the singular instanton homology for the trefoil. This result was already known, since, as shown by Kronheimer and Mrowka, the singular instanton homology of an alternating knot in $S^{3}$ is isomorphic to the reduced Khovanov homology of its mirror [17].

5.2. Unknot in $L(p, 1)$ for $p \bmod 4 \neq 0$. We can make the unknot in $L(p, 1)$ using the mapping class group element $f=T_{a}^{p} \in \mathrm{MCG}_{2}\left(T^{2}\right)$. We find that $L_{d}(\chi, \psi) \cdot f=[(A, B, a, b)]$, where

$$
A=\cos \chi+i \sigma_{z} \sin \chi, \quad B=\cos p \chi+i \sigma_{z} \sin p \chi, \quad a=i \sigma_{x} \cos \psi+i \sigma_{z} \sin \psi, \quad b=a^{-1} .
$$




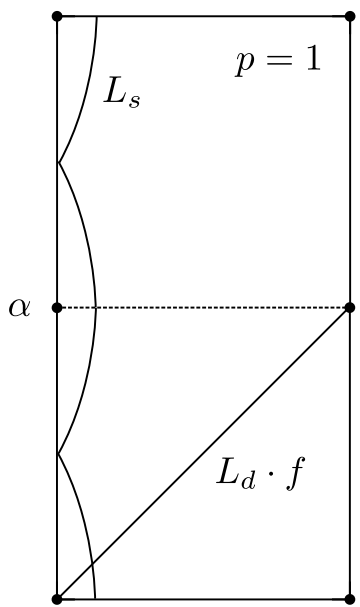

$\beta$

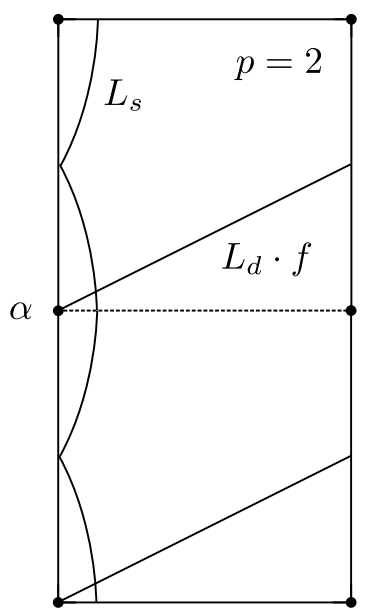

$\beta$

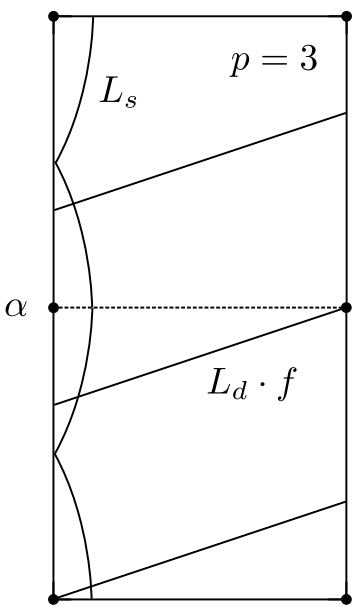

$\beta$

Figure 12. The unknot in $L(p, 1)$, with $f=T_{a}^{p}$, for $p=1,2,3$. The space depicted is the pillowcase $P_{3} \cap\{\gamma=0\}$. Shown are the sphere Lagrangian $L_{s}$ and the disk Lagrangian $L_{d} \cdot f$.

So $L_{d} \cdot f$ is entirely contained in the piece $P_{3}$, and is parameterized by

$$
\alpha\left(L_{d}(\chi, \psi) \cdot f\right)=\chi, \quad \beta\left(L_{d}(\chi, \psi) \cdot f\right)=p \chi,
$$

$$
\gamma\left(L_{d}(\chi, \psi) \cdot f\right)=\psi
$$

Comparing with the parameterization of $L_{s}$ in $P_{3}$ given in equations 81) and (82), we find that the intersection of $L_{d} \cdot f$ with $L_{s}$ in fact takes place in the pillowcase $P_{3} \cap\{\gamma=0\}$. In particular, for each intersection point we have that $\psi=0$ and $\theta \in\{0, \pi\}$. In Figure 12 we plot the intersection of $L_{d} \cdot f$ with $L_{s}$ in the pillowcase $P_{3} \cap\{\gamma=0\}$ for $p=1,2,3$. We find that if $p$ is not a multiple of 4 then we obtain a generating set with $p$ generators. (If $p$ is a multiple of 4 then $\left(L_{d} \cdot f\right) \cap L_{s}$ contains the double-point $(\alpha, \beta, \gamma)=(\pi / 2,0,0)$ of $L_{s}$, and thus our scheme for counting generators fails.)

We will now show that the intersection is transverse at each intersection point. Define functions

$$
h_{1}([\rho])=\operatorname{tr} A a, \quad h_{2}([\rho])=\operatorname{tr} B a .
$$

We find that

$$
\begin{aligned}
& h_{1}\left(L_{s}(\phi, \theta)\right)=\frac{2 \sin (\phi+\nu) \sin \theta}{\sqrt{\cos ^{2} \nu+\sin ^{2} \nu \sin ^{2} \theta}}, \\
& h_{1}\left(L_{d}(\chi, \psi) \cdot f\right)=-2 \sin \chi \sin \psi, \\
& h_{2}\left(L_{s}(\phi, \theta)\right)=0 \text {, } \\
& h_{2}\left(L_{d}(\chi, \psi) \cdot f\right)=-2 \sin p \chi \sin \psi .
\end{aligned}
$$

Thus at each intersection point

$$
\begin{array}{llll}
\partial_{\phi} h_{1}\left(L_{s}(\phi, \theta)\right)=0, & \partial_{\theta} h_{1}\left(L_{s}(\phi, \theta)\right) \neq 0, & \partial_{\chi} h_{1}\left(L_{d}(\chi, \psi) \cdot f\right)=0, & \partial_{\psi} h_{1}\left(L_{d}(\chi, \psi) \cdot f\right) \neq 0, \\
\partial_{\phi} h_{2}\left(L_{s}(\phi, \theta)\right)=0, & \partial_{\theta} h_{2}\left(L_{s}(\phi, \theta)\right)=0, & \partial_{\chi} h_{2}\left(L_{d}(\chi, \psi) \cdot f\right)=0, & \partial_{\psi} h_{2}\left(L_{d}(\chi, \psi) \cdot f\right) \neq 0 .
\end{array}
$$

Equations (81), (82), 164), 168), and 169, together with Figure 12, show that the intersection is transverse at each intersection point.

Remark 5.2. For the case $p=1$ we have that $L(p, 1)=S^{3}$, and our results imply that the unknot in $S^{3}$ has a generating set with a single generator. Since there is a single generator, this amounts to a calculation of the actual singular instanton homology.

Remark 5.3. It is interesting to note that for the unknot $U$ in the lens space $Y=L(p, q)$, the knot Floer homology $\widehat{H F K}(Y, U)$ has rank $p$ (see [1]). 
5.3. Simple knot in $L(p, 1)$ in homology class $1 \in \mathbb{Z}_{p}=H_{1}(L(p, 1) ; \mathbb{Z})$.

Definition 5.1. A knot $K$ in a lens space $L(p, q)$ is said to be simple if the lens space has a Heegaard splitting into solid tori $U_{1}$ and $U_{2}$ with meridian disks $D_{1}$ and $D_{2}$ such that $D_{1}$ intersects $D_{2}$ in $p$ points and $K \cap U_{i}$ is an unknotted arc in disk $D_{i}$ for $i=1,2$ (see [11]).

Remark 5.4. There is exactly one simple knot in each nonzero homology class of $H_{1}(L(p, q) ; \mathbb{Z})=\mathbb{Z}_{p}$ (see [11).

Remark 5.5. The lens space $L(p, 1)$ is a circle bundle over $S^{2}$, and a loop that winds $n$ times around a circle fiber is a simple knot in homology class $n \in \mathbb{Z}_{p}=H_{1}(L(p, 1) ; \mathbb{Z})$.

For $p \geq 2$ we can make the simple knot in $L(p, 1)$ corresponding to the homology class $1 \in \mathbb{Z}_{p}=$ $H_{1}(L(p, 1) ; \mathbb{Z})$ by taking the element of the mapping class group to be $f=\alpha_{1}^{-1} T_{a}^{p} \in \mathrm{MCG}_{2}\left(T^{2}\right)$. We first prove a result that constrains the possible intersection points of $L_{d} \cdot f$ and $L_{s}$ :

Lemma 5.2. If $L_{d}(\chi, \psi) \cdot f=L_{s}(\phi, \theta)$ then $(\chi, \psi)=\left((n+1 / 2)(\pi / p),(-1)^{n+1}(\pi / 2-\epsilon)\right)$ for $n \in\{0, \cdots, p-1\}$ and $\phi=\pi / 2$.

Proof. Define a function $h_{1}: R\left(T^{2}, 2\right) \cap\{\operatorname{tr} A b \neq 0\} \rightarrow \mathbb{R}$ and functions $h_{2}, h_{3}: R\left(T^{2}, 2\right) \rightarrow \mathbb{R}$ by

$$
h_{1}([\rho])=-\frac{\operatorname{tr} A a}{\operatorname{tr} A b}, \quad h_{2}([\rho])=\operatorname{tr} B a, \quad \quad h_{3}([\rho])=\operatorname{tr} B .
$$

We find that

$$
\begin{array}{lll}
h_{1}\left(L_{s}(\phi, \theta)\right)=\frac{\sin (\phi+\nu)}{\sin (\phi-\nu)}, & h_{1}\left(L_{d}(\chi, \psi) \cdot f\right)=1, \\
h_{2}\left(L_{s}(\phi, \theta)\right)=0, & h_{2}\left(L_{d}(\chi, \psi) \cdot f\right)=-2 \cos p \chi, \\
h_{3}\left(L_{s}(\phi, \theta)\right)=2 \cos \nu, & h_{3}\left(L_{d}(\chi, \psi) \cdot f\right)=-2 \sin p \chi \sin \psi .
\end{array}
$$

We also find that

$$
(\operatorname{tr} A b)\left(L_{d}(\chi, \psi) \cdot f\right)=2 \sin \chi \sin \psi .
$$

From equations (173) and (174) it follows that $\operatorname{tr} A b \neq 0$ at each intersection point, and thus equation (171) implies that $\phi=\pi / 2$. Equation $(172)$ implies that $\chi=(n+1 / 2)(\pi / p)$ for $n=0, \cdots, p-1$. Substituting these expressions for $\phi$ and $\chi$ into equation (173), and using the fact that $\nu=\epsilon \sin \phi$, we find that $\psi=$ $(-1)^{n+1}(\pi / 2-\epsilon)$.

The following is a restatement of Theorem 1.5 from the introduction:

Theorem 5.2. If $K$ is the unique simple knot in the lens space $L(p, 1)$ representing the homology class $1 \in \mathbb{Z}_{p}=H_{1}(L(p, 1) ; \mathbb{Z})$, then the rank of the singular instanton homology of $K$ is at most $p$.

Proof. We will argue that each of the $p$ potential intersection points described by Lemma 5.2 is an actual intersection point. Consider the point $L_{d}(\chi, \psi) \cdot f$ with $(\chi, \psi)=\left((n+1 / 2)(\pi / p),(-1)^{n+1}(\pi / 2-\epsilon)\right)$. We find that $L_{d}(\chi, \psi) \cdot f=[(A, B, a, b)]$, where

$$
A=\cos \chi+i \sigma_{x} \sin \chi, \quad B=\cos \epsilon+i \sigma_{z} \sin \epsilon, \quad a=i \hat{a} \cdot \vec{\sigma}, \quad b=i \hat{b} \cdot \vec{\sigma},
$$

$$
\hat{a}=(-1)^{n}(-\cos \epsilon, \sin \epsilon, 0), \quad \hat{b}=\left((-1)^{n} \cos \epsilon,-\sin \epsilon \cos \eta, \sin \epsilon \sin \eta\right)
$$

and $\eta=(1+n(p+2))(\pi / p)$. Since the coefficient of $i \sigma_{x}$ in $A$ and the coefficient of $i \sigma_{z}$ in $B$ are both nonzero, the point $L_{d}(\chi, \psi) \cdot f$ lies in the piece $P_{4}$. From equations (175) and (176), it follows that

$$
\hat{a}\left(L_{d}(\chi, \psi) \cdot f\right)=(-1)^{n}(-\cos \epsilon, \sin \epsilon, 0), \quad \hat{b}\left(L_{d}(\chi, \psi) \cdot f\right)=\left((-1)^{n} \cos \epsilon,-\sin \epsilon \cos \eta, \sin \epsilon \sin \eta\right) .
$$

From equations $70-77$ for $\hat{a}\left(L_{s}(\phi, \theta)\right)$ and $\hat{b}\left(L_{s}(\phi, \theta)\right)$, it follows that

$$
\phi=\pi / 2, \theta \in(0, \pi): \quad \hat{a}\left(L_{s}(\phi, \theta)\right)=(-\cos \epsilon, \sin \epsilon, 0), \quad \hat{b}\left(L_{s}(\phi, \theta)\right)=(\cos \epsilon,-\sin \epsilon \cos \bar{\theta},-\sin \epsilon \sin \bar{\theta}),
$$

$$
\phi=\pi / 2, \theta \in(\pi, 2 \pi): \quad \hat{a}\left(L_{s}(\phi, \theta)\right)=(\cos \epsilon,-\sin \epsilon, 0), \quad \hat{b}\left(L_{s}(\phi, \theta)\right)=(-\cos \epsilon, \sin \epsilon \cos \bar{\theta},-\sin \epsilon \sin \bar{\theta}),
$$


where $\bar{\theta}$ is defined such that

$$
\cos \bar{\theta}=\frac{\cos ^{2} \epsilon \cos ^{2} \theta-\sin ^{2} \theta}{\cos ^{2} \epsilon \cos ^{2} \theta+\sin ^{2} \theta}, \quad \quad \sin \bar{\theta}=\frac{\cos \epsilon \sin 2 \theta}{\cos ^{2} \epsilon \cos ^{2} \theta+\sin ^{2} \theta} .
$$

It is straightforward to verify that for small enough values of $\epsilon$, the maps $(0, \pi) \rightarrow(0,2 \pi), \theta \mapsto \bar{\theta}$ and $(\pi, 2 \pi) \rightarrow(0,2 \pi), \theta \mapsto \bar{\theta}$ are diffeomorphisms. Thus we can always solve equations $177-179$ to obtain a unique value of $\theta$ such that $L_{s}(\phi, \theta)=L_{d}(\chi, \psi) \cdot f$. (For $n$ even we solve $\bar{\theta}(\theta)=-\eta$ for $\theta$, and for $n$ odd we solve $\bar{\theta}(\theta)=\eta+\pi$ for $\theta$.) We conclude that $L_{s}$ and $L_{d} \cdot f$ intersect in $p$ points.

We will now show that $L_{s}$ intersects $L_{d} \cdot f$ transversely at each of these $p$ points. At each intersection point, we find that

$$
\begin{array}{llll}
\partial_{\phi} h_{1}\left(L_{s}(\phi, \theta)\right) \neq 0, & \partial_{\theta} h_{1}\left(L_{s}(\phi, \theta)\right)=0, & \partial_{\chi} h_{1}\left(L_{d}(\chi, \psi) \cdot f\right)=0, & \partial_{\psi} h_{1}\left(L_{d}(\chi, \psi) \cdot f\right)=0, \\
\partial_{\phi} h_{2}\left(L_{s}(\phi, \theta)\right)=0, & \partial_{\theta} h_{2}\left(L_{s}(\phi, \theta)\right)=0, & \partial_{\chi} h_{2}\left(L_{d}(\chi, \psi) \cdot f\right) \neq 0, & \partial_{\psi} h_{2}\left(L_{d}(\chi, \psi) \cdot f\right)=0, \\
\partial_{\phi} h_{3}\left(L_{s}(\phi, \theta)\right)=0, & \partial_{\theta} h_{3}\left(L_{s}(\phi, \theta)\right)=0, & \partial_{\chi} h_{3}\left(L_{d}(\chi, \psi) \cdot f\right)=0, & \partial_{\psi} h_{3}\left(L_{d}(\chi, \psi) \cdot f\right) \neq 0 .
\end{array}
$$

Equations (181)- 183), together with Theorem 4.5, show that the intersection is transverse at each point.

Remark 5.6. It is interesting to note that for a simple knot $K$ in the lens space $Y=L(p, q)$, the knot Floer homology $\widehat{H F K}(Y, K)$ has rank $p$ (see [1]).

Remark 5.7. For the case $p=1$, the knot we have constructed is the unknot in $S^{3}$, and we have have reproduced the result of Section 5.2 for this knot.

Remark 5.8. For the case $p=0$, the knot we have constructed is $K=S^{1} \times\{p t\}$ in $S^{1} \times S^{2}$, and our above result implies that this knot has a generating set with zero generators. This result holds even in the absence of the perturbation, since there are no homomorphisms $\rho: \pi_{1}\left(S^{1} \times S^{2}-K\right) \rightarrow S U(2)$ that take loops around $K$ to traceless matrices.

\section{ACKNOWLEDGMENTS}

The author would like to express his gratitude towards Ciprian Manolescu for providing invaluable guidance. The author was partially supported by NSF grant number DMS-1708320.

\section{REFERENCES}

[1] M. Abouzaid and C. Manolescu, A sheaf-theoretic model for $S L(2, \mathbb{C})$ Floer homology, preprint, arXiv:1708.00289.

[2] P. Bellingeri, On presentations of surface braid groups, J. Algebra, 274(2004), no. 2, 543-563.

[3] J. Birman, Mapping class groups and their relationship to braid groups, Comm. Pure Appl. Math., 22(1969), 213-238.

[4] D. Boozer, Hecke modifications for rational and elliptic curves, arXiv:1805.11184v1.

[5] A. Cattabriga and M. Mulazzani, (1,1)-knots via the mapping class group of the twice punctured torus, Adv. in Geom., 4(2004), 263-277.

[6] S. Donaldson, Floer Homology Groups in Yang-Mills Theory, Cambridge University Press, New York, 2004.

[7] B. Farb and D. Margalit, A Primer on Mapping Class Groups, Princeton University Press, 2012.

[8] S. Gervais, A finite presentation of the mapping class group of a punctured surface, Topology, 40(2001), 703-725.

[9] H. Goda, H. Matsuda, T. Morifuji, T, Knot Floer homology of (1,1)-knots, Geom. Dedic., 112(2005), $197-214$.

[10] M-E. Hamstrom, The space of homeomorphisms on a torus, Illinois J. Math., 9(1965), 59-65.

[11] M. Hedden, On Floer homology and the Berge conjecture on knots admitting lens space surgeries, Tran.s Amer. Math. Soc., 363(2011), 949-968.

[12] M. Hedden, C. Herald, and P. Kirk, The Pillowcase and Perturbations of Traceless Representations of Knot Groups, Geom. Topol., 18(2014), no. 1, 211-287.

[13] M. Hedden, C. Herald, and P. Kirk, The pillowcase and traceless representations of knot groups II: a Lagrangian-Floer theory in the pillowcase, preprint, arXiv:1501.00028v1.

[14] P. Kronheimer and T. Mrowka, Knots, sutures, and excision, J. Differential Geom., 84(2010), 301-364.

[15] P. Kronheimer and T. Mrowka, Knot homology groups from instantons, J. Topol., 4(2011), 835-918.

[16] P. Kronheimer and T. Mrowka, Khovanov homology is an unknot-detector, Publ. Math. Inst. Hautes Études Sci., 113(2011), 97-208.

[17] P. Kronheimer and T. Mrowka, Filtrations on instanton homology, Quantum Topol., 5(2014) 61-97.

[18] C. Labruére and L. Paris, Presentations for the punctured mapping class groups in terms of Artin groups, Algeb. Geom. Topol., 1(2001), 73-114.

[19] J. P. Serre, Trees, Springer-Verlag, New York, 1980.

[20] N. F. Vargas, Geometry of the moduli of parabolic bundles on elliptic curves, preprint, arXiv:1611.05417.

[21] A. Weil, Remarks on the cohomology of groups, Ann. of Math., 2(1964) 149-157. 